\title{
IDEMPOTENT-SEPARATING EXTENSIONS OF REGULAR SEMIGROUPS
}

\author{
A. TAMILARASI \\ Received 1 July 2004 and in revised form 10 March 2005
}

For a regular biordered set $E$, the notion of $E$-diagram and the associated regular semigroup was introduced in our previous paper (1995). Given a regular biordered set $E$, an $E$-diagram in a category $C$ is a collection of objects, indexed by the elements of $E$ and morphisms of $C$ satisfying certain compatibility conditions. With such an $E$-diagram $A$ we associate a regular semigroup $\operatorname{Reg}_{E}(\mathbf{A})$ having $E$ as its biordered set of idempotents. This regular semigroup is analogous to automorphism group of a group. This paper provides an application of $\operatorname{Reg}_{E}(\mathbf{A})$ to the idempotent-separating extensions of regular semigroups. We introduced the concept of crossed pair and used it to describe all extensions of a regular semigroup $S$ by a group $E$-diagram $A$. In this paper, the necessary and sufficient condition for the existence of an extension of $S$ by $A$ is provided. Also we study cohomology and obstruction theories and find a relationship with extension theory for regular semigroups.

\section{Introduction}

If $\pi: T \rightarrow S$ is an idempotent-separating surjective homomorphism of regular semigroups, then the kernel of $\pi$ defines a group $E(S)$-diagram $\mathbf{A}: \underline{C}(E(S)) \rightarrow \mathbf{G R}$ that factors through $\mathbf{D}(B(E(S)))$ and $\pi$ induces an idempotent-separating homomorphism $\Psi$ : $S \rightarrow\left(\operatorname{Reg}_{E(S)}(\mathbf{A})\right) / \operatorname{Inn}_{E(S)}(\mathbf{A})((T, \pi)$ is called an extension of $S$ by the group $E(S)$-diagram A with abstract kernel $\Psi$ ). In this paper, we discuss the following extension problem for regular semigroups.

Given $\Psi: S \rightarrow\left(\operatorname{Reg}_{E(S)}(\mathbf{A})\right) / \operatorname{Inn}_{E(S)}(\mathbf{A})$, find all extensions of $S$ by $A$ with abstract kernel $\Psi$. Of course, given $\Psi$, is it possible that no extension of $S$ by $A$ with abstract kernel $\Psi$ can exist. In this connection, an obstruction theory is developed for finding extensions of $S$ by $A$ which induce the given $\Psi$.

In Section 1, we introduce the concept of a crossed pair and use it to describe all extensions of $S$ by a group $E(S)$-diagram A. In Section 2, we associate with each $\Psi: S \rightarrow$ $\operatorname{Reg}_{E(S)}(\mathbf{A}) / \operatorname{Inn}_{E(S)}(\mathbf{A})$ a three-dimensional cohomology class in the Leech cohomology of $S^{I}$. We show in Theorem 3.6 that the vanishing of this cohomology class is necessary and sufficient condition for the existence of an extension of $S$ by $A$ with abstract kernel $\Psi$. 
We further show that if $\Psi$ has an extension, then the set of all equivalence classes of extensions of $S$ by $\mathbf{A}$ with abstract kernel $\Psi$ is in bijective correspondence with the set of all elements of certain second cohomology group.

Before proceeding further, let us recall some known definitions and results.

For any regular semigroup $S$, we denote by $E(S)$ the set of idempotents of $S$ and by $V(x)$ the set of inverses of an element $x \in S$. Thus $V(x)=\left\{x^{\prime} \in S: x x^{\prime} x=x, x^{\prime} x x^{\prime}=x^{\prime}\right\}$. A pair of elements $\left(x, x^{\prime}\right)$ such that $x^{\prime} \in V(x)$ is called a regular pair in $S$.

A homomorphism $\theta: T \rightarrow S$ of regular semigroups is called idempotent-separating if $\theta$ is one-to-one on the idempotents of $T$. A congruence $\rho$ is called idempotent-separating if the associated projection homomorphism is idempotent-separating. Let $\rho$ be an idempotent-separating congruence on $S$. Then $\rho \subseteq \mathbf{H}$. For each $e \in E(S)$, let $\mathbf{K}_{e}=\rho(e)=\{x \in S$ : $x \rho e\}$. Then $\mathbf{K}_{e}$ is a subgroup of the maximal subgroup $\mathbf{H}_{e}$ of $S$. The family $\mathbf{K}=\left\{\mathbf{K}_{e}: e \in\right.$ $E(S)\}$, where $\left(\mathbf{K}_{S}\right)_{e}=\left\{a \in \mathbf{H}_{e}: a f=f a\right.$ for each idempotent $\left.f \leq e\right\}$.

Definition 1.1 [7]. Let $S$ be a regular semigroup. For each $e \in E(S)$, let $\mathbf{K}_{e}$ be a subgroup of $\mathbf{H}_{e}$. Then $\mathbf{K}=\left\{\mathbf{K}_{e}: e \in E(S)\right\}$ is called a group kernel normal system of $S$ if it satisfies

(i) $a f=f a$ for all $a \in \mathbf{K}_{e}$ and for all $f \in E(S)$ such that $f \leq e$,

(ii) $x^{\prime} \mathbf{K}_{x x^{\prime}} x \subseteq \mathbf{K}_{x^{\prime} x}$ for each regular pair $\left(x, x^{\prime}\right)$ of $S$.

Proposition 1.2 [3]. Let $S$ be a regular semigroup. Let $\mathbf{K}=\left\{\mathbf{K}_{e}: e \in E(S)\right\}$ be a group kernel normal system of S. Define

$$
\begin{gathered}
\rho_{K}=\left\{(x, y) \in S \times S: \text { for some } x^{\prime} \in V(x) \text { and } y^{\prime} \in V(y),\right. \\
\left.x x^{\prime}=y y^{\prime}, x^{\prime} x=y^{\prime} y, \text { and } y^{\prime} x \in \mathbf{K}_{x^{\prime} x}\right\} .
\end{gathered}
$$

Then $\rho_{K}$ is an idempotent-separating congruence on $S$ whose kernel is the group kernel normal system $\mathbf{K}$ of $S$. Conversely, if $\rho$ is an idempotent-separating congruence on $S$, then the kernel $\mathbf{K}$ of $\rho$ is a group kernel normal system of $S$ and $\rho_{K}=\rho$.

Let us recall some results from $[10,13]$.

Let $E$ be a regular biordered set. We write $\omega^{r}=\{(e, f): f e=e\}, \omega^{l}=\{(e, f): e f=e\}$ and $\mathbf{R}=\omega^{r} \cap\left(\omega^{r}\right)^{-1}, \mathbf{L}=\omega^{l} \cap\left(\omega^{l}\right)^{-1}, \omega=\omega^{r} \cap \omega^{l}$.

Definition 1.3. Let $E$ be a regular biordered set and $\mathbf{G}(\mathbf{E})$ the ordered groupoid of $E$-chains of $E$ [13]. The category $\underline{C}(E)$ has objects as the elements of $E$ and a morphism from $e$ to $f$ is a pair $(e, c)$, where $c=c\left(e_{0}, \ldots, e_{n}\right) \in \mathbf{G}(\mathbf{E})$ such that $e \geq e_{0}$ and $e_{n}=f$.

If $(e, c): e \rightarrow f,\left(f, c^{\prime}\right): f \rightarrow g$ are two morphisms, with $c=c\left(e_{0}, \ldots, e_{n}\right), c^{\prime}=c\left(f_{0}, \ldots\right.$, $\left.f_{m}\right)$, then the composite is given by $(e, c)\left(f, c^{\prime}\right)=\left(e,\left(c * f_{0}\right) c^{\prime}\right)$, where $\left(c * f_{0}\right) c^{\prime}$ is the composite of $\left(c * f_{0}\right)$ and $c^{\prime}$ in $\mathbf{G}(\mathbf{E})$. The identity morphism at $e$ is $(e, c(e))$ and the associativity of the composition follows from the transitivity property of the ordered groupoid $\mathbf{G}(\mathbf{E})$ [13, Proposition 3.3].

Throughout this paper, $S$ will denote a regular semigroup with biordered set of idempotents $E=E(S) . B(E)$ the universal regular idempotent generated semigroup on $E$ [13]. $\mathrm{C}(S)$ denotes the category with the set of idempotents $E(S)$ as its objects and morphism from an object $e$ to an object $f$ is a triple $\left(e, x, x^{\prime}\right): e \rightarrow f$, where $\left(x, x^{\prime}\right)$ is a regular pair 
such that $e \geq x x^{\prime}$ and $x^{\prime} x=f$. Composition of morphisms is defined by $\left(e, x, x^{\prime}\right)\left(x^{\prime} x, y\right.$, $\left.y^{\prime}\right)=\left(e, x y, y^{\prime} x^{\prime}\right)$. Define an equivalence relation $\sim$ on the morphisms of $\mathbf{C}(S)$ as follows: if $\left(e, x, x^{\prime}\right),\left(e, y, y^{\prime}\right): e \rightarrow f$ are two morphisms, then $\left(e, x, x^{\prime}\right) \sim\left(e, y, y^{\prime}\right)$ if and only if there exist idempotents $e_{0}, e_{1}, \ldots, e_{n} \in \omega(e)$ with $\left(e_{i-1}, e_{i}\right) \in \mathbf{R} \cup \mathbf{L}, i=1$ to $n$, such that $e_{0}=x x^{\prime}, e_{n}=y y^{\prime}$, and $\left(y, y^{\prime}\right)=\left(e_{n} e_{n-1} \cdots e_{0} x, x^{\prime} e_{0} e_{1} \cdots e_{n}\right)$. Then $\mathbf{D}(S)=\mathbf{C}(S) / \sim$ is the quotient category of $\mathbf{C}(\mathbf{S})$. If we view the underlying groupoid of the inductive groupoid $\mathbf{G}(S)$ of $S$ as a subcategory of $\mathbf{C}(S)$ via the embedding $\left(x, x^{\prime}\right) \rightarrow\left(x x^{\prime}, x, x^{\prime}\right)$, then the evaluation map $\varepsilon_{S}: c\left(e_{0}, e_{1}, \ldots, e_{n}\right) \rightarrow\left(e_{0} e_{1} \cdots e_{n}, e_{n} e_{n-1} \cdots e_{0}\right): \mathbf{G}(E) \rightarrow \mathbf{G}(S)$ extends to a functor $\bar{\varepsilon}_{S}: \underline{C}(E) \rightarrow \mathrm{C}(S)$ such that $\bar{\varepsilon}_{S}(e, c) \rightarrow\left(e, \bar{\varepsilon}_{S}(c)\right)$ for every morphism $(e, c)$ of $\underline{C}(E)$. In particular, by taking $S=B(E)$ we obtain a functor $\bar{\varepsilon}_{B(E)}: \underline{C}(E) \rightarrow \mathrm{C}(B(E))$. By [13, Theorem 6.9], the inclusion $E \subseteq S$ extends uniquely to an idempotent-separating homomorphism $\delta: B(E) \rightarrow S$. If $\mathbf{C}(\delta):\left(e, x, x^{\prime}\right) \rightarrow\left(e, x \delta, x^{\prime} \delta\right): \mathbf{C}(B(E)) \rightarrow \mathbf{C}(S)$ is the induced functor, then $\mathbf{C}(\delta) \bar{\varepsilon}_{B(E)}=\bar{\varepsilon}_{S}$.

If $\theta: S \rightarrow S^{\prime}$ is a homomorphism of regular semigroups, then the maps $e \rightarrow e \theta$; $[e, x$, $\left.x^{\prime}\right] \rightarrow\left[e \theta, x \theta, x^{\prime} \theta\right]$ define a functor $\mathbf{D}(\theta): \mathbf{D}(S) \rightarrow \mathbf{D}\left(S^{\prime}\right)$. Let $E$ be a biordered set. For each $e \in E$, the inclusion $\omega(e) \subseteq E$ induces a functor $\mathbf{D}(B(\omega(e))) \rightarrow \mathbf{D}(B(E))$. Let $\left[e, x, x^{\prime}\right]$, $\left[e, y, y^{\prime}\right]: e \rightarrow f$ be two morphisms of $\mathbf{D}(B(E))$, then

$$
\left[e, x, x^{\prime}\right]=\left[e, y, y^{\prime}\right] \quad \text { if } x=y \text { or } x^{\prime}=y^{\prime} .
$$

Lemma 1.4. Let $\left[e, x, x^{\prime}\right]: e \rightarrow g$ be any morphism of $\mathbf{D}(B(\omega(e)))$ with domain $e$. Then $[e, x$, $\left.x^{\prime}\right]=[e, g, g]$ in $\mathbf{D}(B(\omega(e)))$ and hence in $\mathbf{D}(B(E))$.

Let $S$ be a regular semigroup. Let $\rho_{S}$ be the maximum idempotent-separating congruence on $S$. Then the kernel $\mathbf{K}_{S}$ of $\rho_{S}$ defines a group-valued functor $\mathbf{K}_{S}: \mathbf{C}(S) \rightarrow \mathbf{G R}$, where GR denotes the category of groups, which associates to each object $e$ of $\mathbf{C}(S)$ the group $\left(\mathbf{K}_{S}\right)_{e}$ and to each morphism $\left(e, x, x^{\prime}\right): e \rightarrow f$ the group homomorphism $\mathbf{K}_{S}\left(e, x, x^{\prime}\right)$ : $\left(\mathbf{K}_{S}\right)_{e} \rightarrow\left(\mathbf{K}_{S}\right)_{e}$ given by $(a) \mathbf{K}_{S}\left(e, x, x^{\prime}\right)=x^{\prime} a x$.

Proposition 1.5. If $\rho$ is an idempotent-separating congruence on $S$, then $K^{\rho}: \mathbf{C}(S) \rightarrow \mathbf{G R}$ defined by $K^{\rho}(e)=\rho(e) ; K^{\rho}\left(e, x, x^{\prime}\right)=\mathbf{K}_{S}\left(e, x, x^{\prime}\right) / K^{\rho}(e)$ is a subfunctor of $\mathbf{K}_{S}$. Conversely, if $\mathbf{K}^{\prime}: \mathbf{C}(S) \rightarrow \mathbf{G R}$ is a subfunctor of $\mathbf{K}_{S}$, then $\mathbf{K}^{\prime}=\left\{\mathbf{K}_{e}^{\prime}: e \in E(S)\right\}$ is a group kernel normal system of $S$ and defines, by (1.1), an idempotent-separating congruence $\rho_{K^{\prime}}$ on $S$. Further $\rho \rightarrow \mathbf{K}^{\rho}$ defines a bijective correspondence between the idempotent-separating congruences on $S$ and the subfunctors of $\mathbf{K}_{S}$.

Let $\pi: T \rightarrow S$ be an idempotent-separating homomorphism from $T$ onto $S$. Then $\mathbf{K}^{\pi \pi-1}: \mathbf{C}(T) \rightarrow$ GR factors through $\mathbf{D}(T)$. That is, there is a functor $\operatorname{Ker} \pi: \mathbf{D}(T) \rightarrow \mathbf{G R}$ such that the diagram

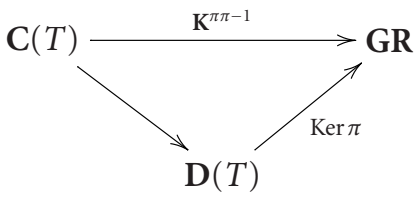

is commutative. Thus $(\operatorname{Ker} \pi)_{e}=\{a \in T: a \pi=e \pi\}, e \in E(T)$, and $a \operatorname{Ker} \pi\left(e, x, x^{\prime}\right)=x^{\prime} a x$, $a \in(\operatorname{Ker} \pi)_{e}$. 
Lemma 1.6 [9, Lemma 4.1]. Let $\pi: T \rightarrow S$ be an idempotent-separating onto homomorphism of regular semigroups. If $t \pi=u \pi=x, t, u \in T, x \in S$, then for each $e \in E(S) \cap \mathbf{L}_{x}$, there exists a unique element $a \in T$ such that $u=t a$ and $a \pi=e$.

Definition 1.7. Let GR be the category of groups. By an E-diagram in GR we mean a functor $\mathbf{A}: \underline{C}(E) \rightarrow \mathbf{G R}$ which factors through $\mathbf{C}(B(E))$. In other words, a functor $\mathbf{A}$ : $\underline{C}(E) \rightarrow \mathbf{G R}$ is an $E$-diagram in $\mathbf{G R}$ if there is a (necessarily unique) functor $\hat{\mathbf{A}}: \mathbf{C}(B(E)) \rightarrow$ GR such that $\mathbf{A}=\hat{\mathbf{A}} \bar{\varepsilon}_{B(E)}$.

Observe that if $\mathbf{A}$ is an $E$-diagram in $\mathbf{G R}$, then for any two morphisms $(e, c),\left(e, c^{\prime}\right)$ : $e \rightarrow f$ in $\underline{C}(E), \mathbf{A}(e, c)=\mathbf{A}\left(e, c^{\prime}\right)$ whenever $\bar{\varepsilon}_{B(E)}(c)=\bar{\varepsilon}_{B(E)}\left(c^{\prime}\right)$.

Let $\mathbf{A}$ be a contravariant $E$-diagram in GR. Then there exists a contravariant functor $\hat{\mathbf{A}}: \mathbf{C}(B(E)) \rightarrow \mathbf{G R}$ such that $\mathbf{A}=\hat{\mathbf{A}} \bar{\varepsilon}_{B(E)}$. For each $e \in E$, let $\mathbf{A}^{e}$ denote the composite

$$
\mathbf{A}^{e}: \underline{C}(\omega(e)) \stackrel{i_{e}}{\longrightarrow} \underline{C}(E) \stackrel{\mathbf{A}}{\longrightarrow} \mathbf{G R} .
$$

Define $\mathbf{G}(\mathbf{A})$ to be the category whose objects are the elements of $E$. A morphism $e \rightarrow f$ is a pair of $(\alpha, \phi)$ consisting of an $\omega$-isomorphism $\alpha: \omega(e) \rightarrow \omega(f)$ and a natural isomorphism $\phi: \mathbf{A}^{e} \rightarrow \mathbf{A}^{f} \underline{C}(\alpha)$, where $\underline{C}(\alpha): \underline{C}(\omega(e)) \rightarrow \underline{C}(\omega(f))$ is the functor defined by the $\omega$-isomorphism $\alpha$, and $\mathbf{A}^{f} \underline{C}(\alpha)$ is the composite

$$
\mathbf{C}(\omega(e)) \stackrel{\mathbf{C}(\alpha)}{\longrightarrow} \underline{C}(\omega(f)) \stackrel{\mathbf{A}^{f}}{\longrightarrow} \mathbf{G R} .
$$

Note that the natural isomorphism $\phi$ assigns to each object $h$ in $\underline{C}(\omega(e))$ an isomorphism $\phi_{h}: \mathbf{A}_{h} \rightarrow \mathbf{A}_{(h) \alpha}$ such that, for any morphism $(h, c): h \rightarrow k$ in $\underline{C}(\omega(e))$, the following diagram commutes:

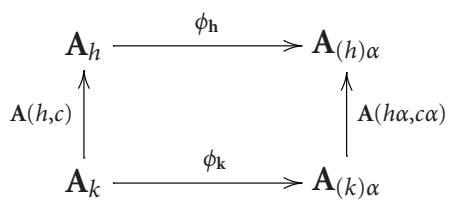

The composite of two morphisms $(\alpha, \phi): e \rightarrow f,(\beta, \Psi): f \rightarrow g$ is given by $(\alpha, \phi)(\beta, \Psi)=$ $(\alpha \beta, \phi(\underline{C}(\alpha) \Psi))$, where $\alpha \beta$ is the composite $\omega(e) \stackrel{\alpha}{\longrightarrow} \omega(f) \stackrel{\beta}{\longrightarrow} \omega(g)$ and the natural isomorphism $\phi(\underline{C}(\alpha) \Psi): \mathbf{A}^{e} \rightarrow \mathbf{A}^{g} \underline{C}(\alpha \beta)$ is defined by

$$
(\phi(\underline{C}(\alpha) \Psi))_{h}=\phi_{h} \circ \Psi_{(h) \alpha}: \mathbf{A}_{h} \longrightarrow \mathbf{A}_{(h) \alpha \beta}
$$

for all $h \in \omega(e)$. For each object $e,\left(1_{e}, \mathbf{1}_{e}\right): e \rightarrow e$, where $1_{e}: \omega(e) \rightarrow \omega(e)$ is the identity $\omega$-isomorphism and $\mathbf{1}_{e}: \mathbf{A}^{e} \rightarrow \mathbf{A}^{e}$ is the identity isomorphism, is the identity morphism of $e$. For an $E$-chain $c=c\left(e_{0}, e_{1}, \ldots, e_{n}\right) \in \mathbf{G}(E)$, define $\varepsilon: \mathbf{G}(E) \rightarrow \mathbf{G}(\mathbf{A})$ by $\varepsilon(c)=\left(\alpha^{c}, \phi^{c}\right)$, 
where $\alpha^{c}: \omega\left(e_{0}\right) \rightarrow \omega\left(e_{n}\right)$ and $\phi^{c}: \mathbf{A}^{e_{0}} \rightarrow \mathbf{A}^{e_{n}} \underline{C}\left(\alpha^{c}\right)$ are such that

$$
(h) \alpha^{c}=(h) \tau_{E}(c)=\tau\left(e_{0}, e_{1}\right) \tau\left(e_{1}, e_{2}\right) \cdots \tau\left(e_{n-1}, e_{n}\right),
$$

$\tau_{E}: \mathbf{G}(E) \rightarrow T^{*}(E)$ is the evaluation map of the inductive groupoid $T^{*}(E)$ of $\omega$ isomorphism of $E$, and $\phi_{h}{ }^{c}=\mathbf{A}(h, h * c)^{-1}: \mathbf{A}_{h} \rightarrow \mathbf{A}_{(h) \alpha}{ }^{c}$ for every $h \in \omega\left(e_{0}\right)$. By [10], it follows that $(\mathbf{G}(\mathbf{A}), \varepsilon)$ is an inductive groupoid.

Let $\mathbf{A}: \underline{C}(E) \rightarrow \mathbf{G R}$ be an $E$-diagram in $\mathbf{G R}$. Let $\operatorname{Reg}_{E}(\mathbf{A})$ be the quotient of $\mathbf{G}(\mathbf{A})$ by the equivalence relation $\rho$, where for any two morphisms $(\alpha, \phi): e \rightarrow f,(\beta, \Psi): f \rightarrow g$ in $\mathbf{G}(\mathbf{A})$,

$$
(\alpha, \phi) \rho(\beta, \Psi) \Longleftrightarrow e \mathbf{R} g, \quad f \mathbf{L} h, \varepsilon(c(e, g))(\beta, \Psi)=(\alpha, \phi) \varepsilon(c(f, h)) .
$$

Also if $[\alpha, \phi],[\beta, \Psi]$ are the elements of $\operatorname{Reg}_{E}(\mathbf{A})$ with representatives $(\alpha, \phi): e \rightarrow f$, $(\beta, \Psi): f \rightarrow g$, then as in $[13]$

$$
[\alpha, \phi][\beta, \Psi]=\left[(\alpha, \phi) \circ_{1}(\beta, \Psi)\right]=\left[((\alpha, \phi) * f l)\left(\alpha^{c}, \phi^{c}\right)(\lg *(\beta, \Psi))\right],
$$

where $l \in S(f, g)$, the sandwich set of $f$ and $g$, and $c=c(f l, l, l g)$. Also note that $E\left(\operatorname{Reg}_{E}(\mathbf{A})\right)=\left[1_{e}, \mathbf{1}_{e}\right]$.

LEMmA 1.8. Let $([x],[y])$ be a regular pair in S such that $[x][y]=\left[1_{e}\right]$ and $[y][x]=\left[1_{f}\right]$. Then there exists $z: e \rightarrow f$ in the inductive groupoid $\mathbf{G}$ such that $[z]=[x]$ and $\left[z^{-1}\right]=[y]$.

\section{Idempotent-separating extensions of regular semigroups}

Consider a regular semigroup $T$ and an idempotent-separating homomorphism $\pi: T \rightarrow$ $S$ of $T$ onto $S$. Let $\operatorname{Ker} \pi: \mathbf{D}(T) \rightarrow$ GR be the group-valued functor defined by the kernel of $\pi$. The inverse $i=(\pi / E(T))^{-1}: E \rightarrow E(T)$ of the biorder isomorphism $\pi / E(T): E(T) \rightarrow$ $E(S)=E$ extends to an idempotent-separating homomorphism î $: B(E) \rightarrow T$ by $[13$, Theorem 6.9] and hence induces a functor $\mathbf{D}(\hat{\mathbf{1}}): \mathbf{D}(B(E)) \rightarrow \mathbf{D}(T)$. If $\pi_{1}: \underline{C}(E) \rightarrow \mathbf{D}(B(E))$ denotes the functor

$$
\left(e, c\left(e_{0}, \ldots, e_{n}\right)\right) \longrightarrow\left[e, e_{0} e_{1} \cdots e_{n}, e_{n} e_{n-1} \cdots e_{0}\right]
$$

then the composite

$$
\mathbf{A}^{\pi}=\operatorname{Ker} \pi \mathbf{D}(\hat{\mathbf{1}}) \pi_{1}: \underline{C}(E) \longrightarrow \mathbf{G R}
$$

is a group $E$-diagram which factors through $\mathbf{D}(B(E))$. Thus $\mathbf{A}_{e}{ }^{\pi}=\{t \in T: t \pi=e\}$ for each object $e$ of $\underline{C}(E)$ and

$$
A^{\pi}\left(e, c\left(e_{o}, \ldots, e_{n}\right)\right)=\operatorname{Ker} \pi\left[e i,\left(e_{o} i\right) \cdots\left(e_{n} i\right),\left(e_{n} i\right) \cdots\left(e_{o} i\right)\right]: \mathbf{A}_{e} \longrightarrow \mathbf{A}_{f}
$$

for each morphism $\left(e, c\left(e_{o}, \ldots, e_{n}\right)\right): e \rightarrow f$ of $\underline{C}(E)$. This observation motivates the following. 
Definition 2.1. Let $\mathbf{A}: \underline{C}(E) \rightarrow$ GR be a (covariant) group $E$-diagram that factors through $\mathbf{D}(B(E))$. An extension of the regular semigroup $S$ by the group $E$-diagram $\mathbf{A}$ is a triple $\varepsilon_{T}=$ $(T, \pi, U)$ consisting of a regular semigroup $T$, an idempotent-separating homorphism $\pi: T \rightarrow S$ of $T$ onto $S$, and a natural isomorphism of functors $U: \mathbf{A} \rightarrow \mathbf{A}^{\pi}$.

Remark 2.2. Let $e \in E$ and let $\mathbf{A}^{e}$ be the composite $\underline{C}(\omega(e)) \stackrel{i_{e}}{\rightarrow} \underline{C}(E) \stackrel{\mathbf{A}}{\rightarrow}$ GR. For each $x \in \mathbf{A}_{e}$, we define a natural isomorphism $\eta^{x}: \mathbf{A}^{e} \rightarrow \mathbf{A}^{e}$ as follows. Given $h \in \omega(e)$, let $x_{h}=(x) \mathbf{A}(e, h) \in \mathbf{A}_{h}$, and let $\eta_{h}{ }^{x}: \mathbf{A}_{h} \rightarrow \mathbf{A}_{h} ;(a) \eta_{h}{ }^{x}=x_{h}{ }^{-1} a x_{h}$ be the inner automorphism defined by $x_{h}$. If $m=\left(h, c\left(h_{0}, h_{1}, \ldots, h_{n}\right)\right): h \rightarrow k$ is a morphism of $\underline{C}(\omega(e))$, then

$$
x_{h} \mathbf{A}(m)=(x) \mathbf{A}(e, h) \mathbf{A}(m)=(x) \mathbf{A}((e, h) m)=(x) \mathbf{A}(e, k)=x_{k}
$$

and therefore the diagram

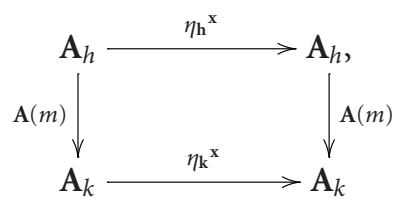

is commutative. Thus the map $h \rightarrow \eta_{h}{ }^{x}, h \in \omega(e)$, defines a natural isomorphism $\eta^{x}$ : $\mathbf{A}^{e} \rightarrow \mathbf{A}^{e}$. If $\operatorname{Reg}_{E}(\mathbf{A})$ is the regular semigroups of partial isomorphisms of the $E$-diagram $\mathbf{A}$, then $\left[1_{e}, \eta^{x}\right] \in \operatorname{Reg}_{E}(\mathbf{A})$, where $1_{e}: \omega(e) \rightarrow \omega(e)$ is the identity isomorphism. Clearly $\eta^{x} \eta^{y}=\eta^{x y}$ for all $x, y \in \mathbf{A}_{e}$ and hence the map

$$
\eta: x \rightarrow\left[1_{e}, \eta^{x}\right]: \mathbf{A}_{e} \longrightarrow \operatorname{Reg}_{E}(\mathbf{A})
$$

is a homomorphism. Denote the image of $\mathbf{A}_{e}$ under $\eta$ by $\operatorname{Inn}(\mathbf{A})_{e}$. Then $\operatorname{Inn}(\mathbf{A})_{e}$ is a subgroup of the maximal group $\mathbf{H}_{\left[1_{e}, \mathbf{1}_{e}\right]}$ of $\operatorname{Reg}_{E}(\mathbf{A})$. We write

$$
\operatorname{Inn}_{E}(\mathbf{A})=\left\{\operatorname{Inn}_{E}(\mathbf{A})\right\}_{e \in E} .
$$

Proposition 2.3. $\operatorname{Inn}_{E}(\mathbf{A})$ is a group kernel normal system in $\operatorname{Reg}_{E}(\mathbf{A})$.

Proof. Let $x \in \mathbf{A}_{e}$ and $h \in \omega(e)$. Then $\left[1_{e}, \eta^{x}\right]\left[1_{h}, \mathbf{1}_{h}\right]=\left[1_{h}, \eta_{h}^{x}\right]=\left[1_{h}, \mathbf{1}_{h}\right]\left[1_{e}, \eta^{x}\right]$. Next, let $\left(s, s^{\prime}\right)$ be a regular pair in $\operatorname{Reg}_{E}(\mathbf{A})$ such that $s s^{\prime}=\left[1_{e}, \mathbf{1}_{e}\right]$ and $s^{\prime} s=\left[1_{f}, \mathbf{1}_{f}\right]$. Using Lemma 1.8, choose a morphism $(\alpha, \phi): e \rightarrow f$ in $G(A)$ such that $[\alpha, \phi]=s,\left[\alpha^{-1}, \phi^{-1}\right]=s^{\prime}$. Then, for any $x \in \mathbf{A}_{e}$, we have $s^{\prime}(x) \eta s=\left[\alpha^{-1}, \phi^{-1}\right]\left[1_{e}, \eta^{x}\right][\alpha, \phi]=\left[1_{f}, \eta^{(x) \phi}{ }_{e}\right]=\left((x) \phi_{e}\right) \eta \in$ $\operatorname{Inn}(\mathbf{A})_{f}$. Hence, by Definition 1.1, $\operatorname{Inn}_{E}(\mathbf{A})$ is a group kernel normal system in $\operatorname{Reg}_{E}(\mathbf{A})$.

Let $\operatorname{Reg}_{E}(\mathbf{A}) / \operatorname{Inn}_{E}(\mathbf{A})$ be the quotient of $\operatorname{Reg}_{E}(\mathbf{A})$ by the idempotent-separating congruence determined by $\operatorname{Inn}_{E}(\mathbf{A})$ (see Proposition 1.2) and let

$$
t: \operatorname{Reg}_{E}(\mathbf{A}) \longrightarrow \operatorname{Reg}_{E}(\mathbf{A}) / \operatorname{Inn}_{E}(\mathbf{A})
$$

be the associated projection homomorphism. 
We next define the centre of the E-diagram A. For any $e \in E$, let

$$
\begin{aligned}
\mathbf{Z}(\mathbf{A})_{e} & =\operatorname{Ker}\left\{\eta: \mathbf{A}_{e} \longrightarrow \operatorname{Reg}_{E}(\mathbf{A})\right\}=\left\{a \in \mathbf{A}_{e}:(a) \eta=\left[1_{e}, \mathbf{1}_{e}\right]\right\} \\
& =\left\{a \in \mathbf{A}_{e}:(a) A(e, h)=a_{h} \in \mathbf{Z}\left(\mathbf{A}_{h}\right) \text { for every } h \in \omega(e)\right\} .
\end{aligned}
$$

Evidently $\mathbf{Z}(\mathbf{A})_{e}$ is an abelian normal subgroup of $\mathbf{A}_{e}$. If $\left(e, c=c\left(e_{0}, \ldots, e_{n}\right)\right): e \rightarrow f$ is a morphism in $\underline{C}(E)$, then $\left(\mathbf{Z}(\mathbf{A})_{e}\right) \mathbf{A}(e, c) \subseteq \mathbf{Z}(\mathbf{A})_{f}$. For, if $a \in \mathbf{Z}(\mathbf{A})_{e}$, then for any element $h \in \omega(f)$, letting $c^{\prime}=c * h=c\left(h_{0}, h_{1}, \ldots, h_{n}\right)$, we have $(a) \mathbf{A}(e, c) \mathbf{A}(f, h)=(a) \mathbf{A}((e, c)(f$, $h))=(a) \mathbf{A}(e, c * h)=(a) \mathbf{A}\left(e, c^{\prime}\right)=(a) \mathbf{A}\left(e, h_{0}\right) \mathbf{A}\left(h_{0}, c^{\prime}\right) \in \mathbf{Z}(\mathbf{A})_{h}$, since $(a) \mathbf{A}\left(e, h_{0}\right) \in \mathbf{Z}(\mathbf{A})_{h 0}$ and $\mathbf{A}\left(h_{0}, c^{\prime}\right): \mathbf{A}_{h 0} \rightarrow \mathbf{A}_{h}$ is an isomorphism of groups. Therefore, the maps

$$
e \longrightarrow \mathbf{Z}(\mathbf{A})_{e} ; \quad(e, c) \longrightarrow \mathbf{A}(e, c) \mid \mathbf{Z}(\mathbf{A})_{e}: \mathbf{Z}(\mathbf{A})_{e} \longrightarrow \mathbf{Z}(\mathbf{A})_{f}
$$

define a functor $\mathbf{Z}(\mathbf{A}): \underline{C}(E) \rightarrow \mathbf{G R}$, which is a subfunctor of $\mathbf{A}: \underline{C}(E) \rightarrow \mathbf{G R}$. Since $\mathbf{Z}(\mathbf{A})_{e}$ 's are abelian groups, we may also view $\mathbf{Z}(\mathbf{A})$ as a functor from $\underline{C}(E)$ to $\mathbf{A b}$, the category of abelian groups.

Definition 2.4. The functor $\mathbf{Z}(\mathbf{A}): \underline{C}(E) \rightarrow \mathbf{A b}$ is called the centre of $\mathbf{A}$.

Proposition 2.5. The sequence

$$
1 \longrightarrow \mathbf{Z}(\mathbf{A}) \stackrel{i}{\longrightarrow} \mathbf{A} \stackrel{\eta}{\longrightarrow} \operatorname{Reg}_{E}(\mathbf{A}) \stackrel{t}{\longrightarrow} \operatorname{Reg}_{E}(\mathbf{A}) / \operatorname{Inn}_{E}(\mathbf{A}) \longrightarrow 1
$$

is exact in the sense that $t$ is an idempotent-separating onto homomorphism and the sequence

$$
1 \longrightarrow \mathbf{Z}(\mathbf{A})_{e} \stackrel{i e}{\longrightarrow} \mathbf{A}_{e} \longrightarrow(\operatorname{Ker} t)_{e} \longrightarrow 1
$$

is an exact sequence of groups for each $e \in E$.

Since $\mathbf{A}$ factors through $\mathbf{D}(B(E))$, so is the centre $\mathbf{Z}(\mathbf{A}): \underline{C}(E) \rightarrow \mathbf{A b}$. Let $\operatorname{Reg}_{E}(Z(\mathbf{A}))$ be the regular semigroup of partial isomorphisms of $\mathbf{Z}(\mathbf{A})$. If $(\alpha, \phi): e \rightarrow f$ is a morphism in $\mathbf{G}(\mathbf{A})$, then for each $h \in \omega(e), \phi_{h}: \mathbf{A}_{h} \rightarrow \mathbf{A}_{(h) \alpha}$ induces by restriction an isomorphism $\bar{\phi}_{h}$ : $Z(\mathbf{A})_{h} \rightarrow Z(\mathbf{A})_{(h) \alpha}$ and therefore the map $h \rightarrow \bar{\phi}_{h}$ defines a natural $\bar{\phi}: \mathbf{Z}(\mathbf{A})^{e} \rightarrow \mathbf{Z}(\mathbf{A})^{f} \underline{C}(\alpha)$. Thus we have an idempotent-separating homomorphism

$$
u: \operatorname{Reg}_{E}(\mathbf{A}) \longrightarrow \operatorname{Reg}_{E} \mathbf{Z}((\mathbf{A}))
$$

defined by $[\alpha, \phi] u=[\alpha, \bar{\phi}]$, for $[\alpha, \phi] \in \operatorname{Reg}_{E}(\mathbf{A})$. If $x \in \mathbf{A}_{e}$, then clearly $\bar{\eta}^{x}: \mathbf{Z}(\mathbf{A})^{e} \rightarrow \mathbf{Z}(\mathbf{A})^{e}$ is the identity natural isomorphism. Hence, $u$ induces an idempotent-separating homomorphism

$$
v: \operatorname{Reg}_{E}(\mathbf{A}) / \operatorname{Inn}_{E}(\mathbf{A}) \longrightarrow \operatorname{Reg}_{E} \mathbf{Z}((\mathbf{A}))
$$

such that $t v=u$. 
Definition 2.6. Two extensions $\varepsilon_{T}=(T, \pi, U)$ and $\varepsilon_{T^{\prime}}=\left(T^{\prime}, \pi^{\prime}, U^{\prime}\right)$ of $S$ by A are equivalent if there exists an isomorphism $\theta: T \rightarrow T^{\prime}$ of regular semigroups such that

(i) $\theta \pi^{\prime}=\pi$,

(ii) for each $e \in E$, the diagram

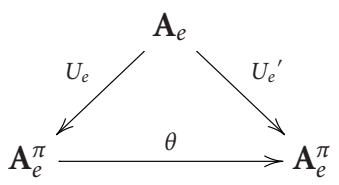

is commutative.

This defines an equivalence relation on any set of extensions of $S$ by $\mathbf{A}$.

Given an extension $\varepsilon_{T}=(T, \pi, U)$ of $S$ by $\mathbf{A}$, we usually identify $\mathbf{A}$ with $\mathbf{A}^{\pi}$ so that $U=\mathbf{1}$, the identity natural isomorphism on $\mathbf{A}$.

Let $\varepsilon_{T}=(T, \pi, \mathbf{1})$ be an extension of $S$ by $\mathbf{A}$ and let $\operatorname{Reg}_{E}(\mathbf{A})$ be the regular semigroup of partial isomorphisms of $\mathbf{A}$. We define a map $\bar{\mu}: T \rightarrow \operatorname{Reg}_{E}(\mathbf{A})$ as follows. Given $x \in T$, choose $x^{\prime} \in V(x)$ and let

$$
(x) \bar{\mu}=\left[\beta\left(x, x^{\prime}\right), \Psi\left(x, x^{\prime}\right)\right],
$$

where the $\omega$-isomorphism $\beta\left(x, x^{\prime}\right): \omega\left(\left(x x^{\prime}\right) \pi\right) \rightarrow \omega\left(\left(x^{\prime} x\right) \pi\right)$ is given by

$$
\text { (h) } \beta\left(x, x^{\prime}\right)=\left(x^{\prime} \pi\right) h(x \pi), \quad h \in \omega\left(\left(x x^{\prime}\right) \pi\right),
$$

and the natural isomorphism $\Psi\left(x, x^{\prime}\right): \mathbf{A}^{\left(x x^{\prime}\right) \pi} \rightarrow A^{\left(x^{\prime} x\right) \pi} \underline{C}\left(\beta\left(x, x^{\prime}\right)\right)$ sends each object $h$ of $\underline{C}\left(\omega\left(x x^{\prime}\right) \pi\right)$ to the isomorphism

$$
\Psi_{h}\left(x, x^{\prime}\right): a \longrightarrow x^{\prime} a x: \mathbf{A}_{h} \longrightarrow \mathbf{A}_{\left(x^{\prime} \pi\right) h(x \pi)} .
$$

The element $(x) \bar{\mu}$ is independent of the chosen $x^{\prime} \in V(x)$, and $x \rightarrow(x) \bar{\mu}$ defines an idempotent-separating homomorphism $\bar{\mu}: T \rightarrow \operatorname{Reg}_{E}(\mathbf{A})$ such that $(e) \bar{\mu}=\left[1_{e \pi}, \mathbf{1}_{e \pi}\right]$ for every $e \in E(T)$. These facts are immediate from [10, Theorem 1.6], as $\bar{\mu}$ is essentially the idempotent-separating homomorphism induced by the composite: $\operatorname{Ker} \pi: \mathbf{D}(T) \rightarrow \mathbf{G R}$ with the projection functor $\mathbf{C}(T) \rightarrow \mathbf{D}(T):\left(e, x, x^{\prime}\right) \rightarrow\left[e, x, x^{\prime}\right]$. If $x \in \mathbf{A}_{e}$ with inverse $x^{-1}$ in $A_{e^{\prime}}$, then from (2.17) and (2.18) we obtain

$$
(x) \bar{\mu}=\left[\beta\left(x, x^{-1}\right), \Psi\left(x, x^{-1}\right)\right]=\left[1_{e}, \eta^{x}\right] \in(\operatorname{Inn} \mathbf{A})_{e},
$$

where $\eta: \mathbf{A}_{e} \rightarrow \operatorname{Reg}_{E}(\mathbf{A})$ is as in (2.6). Hence, $\bar{\mu}$ induces an idempotent-separating homomorphism $\Psi: S \rightarrow \operatorname{Reg}_{E}(\mathbf{A}) / \operatorname{Inn}_{E}(\mathbf{A})$ completing the square

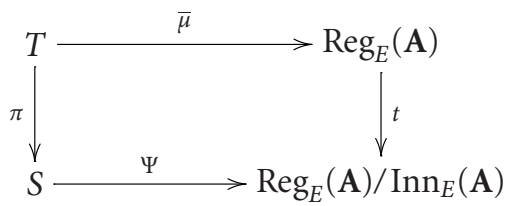


where $t$ is the projection homomorphism. From (2.17) it is clear that the diagram

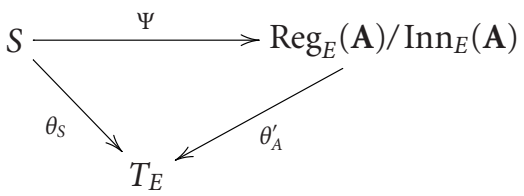

is commutative. Here as in [13], $\theta_{S}$ denotes the fundamental representation of $S$ and $\theta_{A}^{\prime}$ is the idempotent-separating homomorphism induced by the fundamental representation $\theta_{A}: \operatorname{Reg}_{E}(\mathbf{A}) \rightarrow T_{E}:[\alpha, \phi] \mapsto[\alpha]$ of $\operatorname{Reg}_{E}(\mathbf{A})$.

Definition 2.7. Let $\mathbf{A}: \underline{C}(E) \rightarrow \mathbf{G R}$ be a group $E$-diagram that factors through $\mathbf{D}(B(E))$. Let $\Psi: S \rightarrow \operatorname{Reg}_{E}(\mathbf{A}) / \operatorname{Inn}_{E}(\mathbf{A})$ be an idempotent-separating homomorphism such that diagram (2.21) is commutative. Then the triple $(S, \Psi, \mathbf{A})$, or just $\Psi$, is called an abstract kernel.

The discussion preceding Definition 2.7 shows that an extension $\varepsilon_{T}=(T, \pi, \mathbf{1})$ of $S$ by A defines an abstract kernel $\Psi: S \rightarrow \operatorname{Reg}_{E}(\mathbf{A}) / \operatorname{Inn}_{E}(\mathbf{A})$ which we call the abstract kernel of the extension $\varepsilon_{T} . \varepsilon_{T}$ is called an extension of the abstract kernel $\Psi$.

Remark 2.8. If $\Psi: S \rightarrow \operatorname{Reg}_{E}(\mathbf{A}) / \operatorname{Inn}_{E}(\mathbf{A})$ is an abstract kernel, then the following two properties of $\Psi$ are immediate from the commutativity of diagram (2.21):

(i) $\left[1_{e}, \mathbf{1}_{e}\right] \in(e) \Psi$ for every $e \in E$,

(ii) if $[\alpha, \phi] \in(x) \Psi$, then for some $x^{\prime} \in V(x)$, there is a representative $\left(\alpha^{\prime}, \phi^{\prime}\right): x x^{\prime} \rightarrow$ $x^{\prime} x$ of $[\alpha, \phi]$ in the inductive groupoid $\mathbf{G}(\mathbf{A})$ such that $(e) \alpha^{\prime}=x^{\prime} e x$ for all $e \in$ $\omega\left(x x^{\prime}\right)$.

Note that if (ii) holds for one $x^{\prime} \in V(x)$, then it holds for all $x^{\prime} \in V(x)$.

The rest of the section is devoted to a description of extensions of $S$ by $\mathbf{A}$ which induce the given abstract kernel $\Psi$. We first fix some notation and develop necessary preliminaries for this purpose.

Remark 2.9. Suppose $\mathbf{A}$ is a covariant $E$-diagram in an arbitrary category $C$ which factors through $\mathbf{D}(B(E))$. That is, there is a (necessarily unique) functor $\hat{\mathbf{A}}: \mathbf{D}(B(E)) \rightarrow C$ such that the diagram

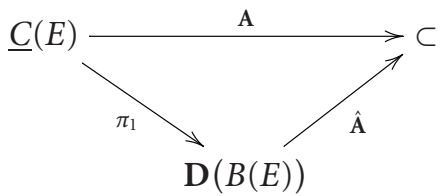

is commutative, where $\pi_{1}:\left(e, c\left(e_{0}, e_{1}, \ldots, e_{n}\right)\right) \rightarrow\left[e, e_{0} e_{1} \cdots e_{n}, e_{n} e_{n-1} \cdots e_{0}\right]: \underline{C}(E) \rightarrow$ $\mathbf{D}(B(E))$ is the composite $\underline{C}(E) \stackrel{\bar{\varepsilon}_{B(E)}}{\longrightarrow} \mathbf{C}(B(E)) \rightarrow \mathbf{D}(B(E))$. In this case, for any idempotent-separating homomorphism $\mu: S \rightarrow \operatorname{Reg}_{E}(\mathbf{A})$ with $\mu \theta_{S}=\theta_{A}$, the associated covariant functor $\mathbf{A}_{\mu}: \mathbf{C}(S) \rightarrow C:\left(\mathbf{A}_{\mu}\right)_{e}=\mathbf{A}_{e} ; e \in \mathbf{C}(S)$ and $\mathbf{A}_{\mu}\left(e, x, x^{\prime}\right)=\left(\phi_{x x^{\prime}}\left(x, x^{\prime}\right)\right)^{-1} \mathbf{A}\left(e, x x^{\prime}\right)$ for each morphism $\left(e, x, x^{\prime}\right): e \rightarrow f$ of $\mathbf{C}(S)$ factors through $\mathbf{D}(S)$. We denote the functor 
2954 Idempotent-separating extensions of regular semigroups

$e \rightarrow \mathbf{A}_{e} ;\left[e, x, x^{\prime}\right] \rightarrow \mathbf{A}\left(e, x x^{\prime}\right) \phi_{x x^{\prime}}\left(x, x^{\prime}\right): \mathbf{D}(S) \rightarrow C$ by $\overline{\mathbf{A}}_{\mu}$ itself so that the diagram

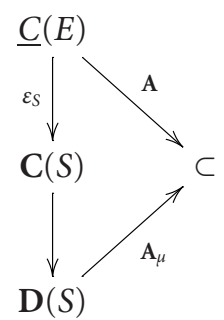

is commutative.

If $e, f \in E$, then for any $h \in S(e, f),(e, c(e h, h, h f))$ is a morphism from $e$ to $h f$ in $\underline{C}(E)$.

We write

$$
D(h, e, f)=(e, c(e h, h, h f)) .
$$

If $f \omega^{l} e$, then we write

$$
L(e, f)=(e, c(e f, f)) .
$$

Note that $L(e, f)=D(f, e, f)$. Also note that

$$
\pi_{1} D(h, e, f)=[e, e f, h]: e \longrightarrow h f, \quad \pi_{1} L(e, f)=[e, e f, f]: e \longrightarrow f,
$$

where $\pi_{1}: \underline{C}(E) \rightarrow \mathbf{D}(B(E))$ is as in Remark 2.9.

Lemma 2.10. Let $\mathbf{A}: \underline{C}(E) \rightarrow \mathbf{G R}$ be an E-diagram that factors through $\mathbf{D}(B(E))$. Let $e, f$, $g, \ldots$ denote arbitrary elements of $E$.

(i) If $g \omega^{l} f \omega^{l} e$, then

$$
\mathbf{A}(L(e, f) L(f, g))=\mathbf{A}(L(e, g))
$$

(ii) If $f \omega^{l} e, h \in S(f, g)$, then $h \in S(e, h g)$ and

$$
\mathbf{A}(L(e, f) D(h, f, g))=\mathbf{A}(D(h, e, h g)) .
$$

If, in addition, $h \in S(e, g)$ (this happens, e.g., $f \mathbf{L} e$ ), then

$$
\mathbf{A}(L(e, f) D(h, f, g))=\mathbf{A}(D(h, e, g)) .
$$

(iii) If $h \in S(e, f)$ and $g \in S(e, k)$, with $k \omega f$, then $g f \in S(h f, k)$ and

$$
\mathbf{A}(D(h, e, f) D(g f, h f, k))=\mathbf{A}(D(g, e, k)) .
$$

(iv) If $h \in S(e, f), g \omega^{l} e, g \omega^{r} f$, then $g \in S(e, g f), g f \omega^{l} h f$, and

$$
\mathbf{A}(D(h, e, f) L(h f, g f))=\mathbf{A}(D(g, e, g f)) .
$$


If, in addition, $g \in S(e, f)$, then

$$
\mathbf{A}(D(h, e, f) L(h f, g f))=\mathbf{A}(D(g, e, f)) .
$$

(v) If $h \in S(e, f), g \mathbf{R} f$, then $h \in S(e, g), h f \in S(h f, g)$, and

$$
\mathbf{A}(D(h, e, f) D(h f, h f, g))=\mathbf{A}(D(h, e, g)) .
$$

(vi) If $f \omega^{l} e, g \in S(e, n), h \in S(f, e g), m \in S(f, n), k \in S(h(e g), n)$, with $m n \mathbf{L} k n$, then

$$
\mathbf{A}(D(h, f, e g) D(k, h(e g), n))=\mathbf{A}(D(m, f, n) L(m n, k n)) .
$$

Proof. By Remark 2.9, it is sufficient to prove (i)-(ii) replacing A by the functor $\pi_{1}$ : $\underline{C}(E) \rightarrow \mathbf{D}(B(E))$. We frequently use (1.2) to prove the lemma.

(i) Using (1.2) we get $\pi_{1}(L(e, f) L(f, g))=\pi_{1}\left(L(e, f) \pi_{1} L(f, g)\right)=[e, e f, f][f, f g, g]=$ $[e,(e f)(f g), g]=[e, e g, g]=\pi_{1}(L(e, g))$. This proves (i).

(ii) Let $f \omega^{l} e$ and $h \in S(f, g)$. Then clearly $h \in S(e, h g)$, and $\pi_{1}(L(e, f) D(h, f, g))=$ $[e, e f, f][f, f g, h]=[e,(e f)(f g), h]=[e, e(h g), h]=\pi_{1}(D(h, e, h g))$. If $h \in S(e, g)$, then $D(h, e, g)=D(h, e, h g)$. Therefore, the second statement follows from the first.

(iii) Clearly $g f \in S(h f, k)$. Now $\pi_{1}(D(h, e, f) D(g f, h f, k))=[e, e f, h][h f,(h f) k, g f]=$ $[e,(e f)((h f) k),(g f) h]=[e, e k, g h]=[e, e k, g]=\pi_{1}(D(g, e, k))$, since $(e f)((h f) k)=e k$, and $(g f) h=g h$.

(iv) Clearly $g \in S(e, g f)$ and $g f \omega^{l} h f$. By taking $k=g f$ in (iii) and observing $D(g f, h f$, $g f)=L(h f, g f)$, we get $\pi_{1}(D(h, e, f) L(h f, g f))=\pi_{1}(D(g, e, g f))$. The last relation follows from this since $D(g, e, g f)=D(g, e, f)$, if $g \in S(e, f)$.

(v) Let $h \in S(e, f), g \mathbf{R} f$. Then, by [13, Proposition 2.12], $S(e, f)=S(e, g)$ and so $h \in$ $S(e, g)$. Clearly $h f \in S(h f, g)$. Further, since $(h f) h=h(f h)=h$, we get $\pi_{1}(D(h, e, f) D(h f$, $h f, g))=[e, e f, h][h f,(h f) g, h f]=[e,(e f)((h f) g), h]=[e, e g, h]=\pi_{1}(D(h, e, g))$.

(vi)

$$
\begin{aligned}
\pi_{1}(D & (h, f, e g) D(k, h(e g), n)) \\
& =[f, f(e g), h][h(e g),(h(e g) n, k)] \\
& =[f,(f(e g))((h(e g)) n), k h] \\
& =[f,(f(e g)) n, k h] \quad \text { since }(e g) h=h,(f h)(e g)=f(e g) \\
& =[f, f n, k h] \quad \text { since }(e g) n=e n, f e=f \\
& =[f, f(n m) n, k h] \quad \text { since } f m n=f n, n m=m \\
& =[f,(f n)(m n), k h] \\
& =[f,(f n)(m n),(k n) m] \\
& =[f, f n, m][m n, m n, k n] \\
& =\pi_{1}(D(m, f, n) L(m n, k n)) .
\end{aligned}
$$

The proof of the lemma is complete. 
We fix once and for all a map $*: S \rightarrow S$ such that

(i) $x^{*} \in V(x)$ for every $x \in S$,

(ii)

$$
x^{*} \in \mathbf{H}_{e} \quad \text { if } x \in \mathbf{H}_{e} .
$$

Suppose $\Psi: S \rightarrow \operatorname{Reg}_{E}(\mathbf{A}) / \operatorname{Inn}_{E}(\mathbf{A})$ is an abstract kernel, and let $\sigma: S \rightarrow \operatorname{Reg}_{E}(\mathbf{A})$ be a map such that $(x) \sigma \in(x) \Psi$, for every $x \in S$. By Remark 2.9(ii) and by [13], each $(x) \sigma$ has a unique representative in $\mathbf{G}(\mathbf{A})$ with domain $x x^{*}$ and range $x^{*} x$. We denote this morphism by $(\alpha(x), \phi(x)): x x^{*} \rightarrow x^{*} x$ so that $[\alpha(x), \phi(x)]=(x) \sigma$; recall by Remark 2.9(ii), (h) $\alpha(x)=x^{*} h x$ for all $h \in \omega\left(x x^{*}\right)$. Using $\sigma$ we will define a biaction of $S$ on the disjoint union

$$
\mathbf{A}=\bigcup_{x \in S} \mathbf{A}_{x}, \quad \text { where } \mathbf{A}_{x}=\mathbf{A}_{x^{*} x}
$$

For $x, y \in S$, define

$$
a \longrightarrow x \cdot a: \mathbf{A}_{y} \longrightarrow \mathbf{A}_{x y}, \quad a \longrightarrow a \cdot x: \mathbf{A}_{y} \longrightarrow \mathbf{A}_{y x}
$$

by

$$
\begin{gathered}
x \bullet a=a \mathbf{A}\left(L\left(y^{*} y,(x y)^{*} x y\right)\right), \\
a \bullet x=a \mathbf{A}\left(D\left(h, y^{*} y, x x^{*}\right)\right) \phi_{h x x^{*}}(x) \mathbf{A}\left(L\left(x^{*} h x,(y x)^{*} y x\right)\right),
\end{gathered}
$$

where $h \in S\left(y^{*} y, x x^{*}\right)$ and $\phi_{h x x^{*}}(x): \mathbf{A}_{h x x^{*}} \rightarrow \mathbf{A}_{\left(h x x^{*}\right) \alpha(x)=x^{*} h x}$ is the component of $\phi(x)$ at $h x x^{*} \in \omega\left(x x^{*}\right)$. If $k \in S\left(y^{*} y, x x^{*}\right)$ is any other element, then the following diagram commutes:

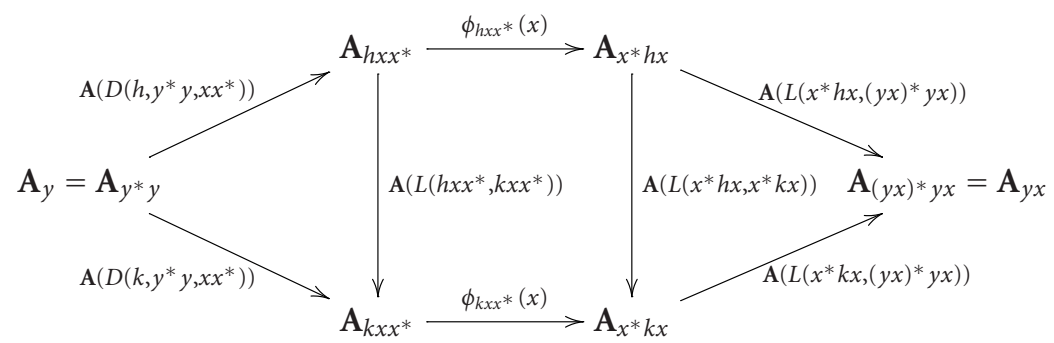

The first triangle is commutative by Lemma 2.10(iv) and the last triangle is commutative by Lemma $2.10(\mathrm{i})$, since $(y x)^{*} y x \omega^{l} x^{*} k x \omega^{l} x^{*} h x$. Finally the commutativity of the rectangle follows from the naturality of $\phi(x)$. Hence, $a \bullet x$ does not depend on the choice of $h$. Clearly $a \rightarrow a \bullet x$ and $a \rightarrow x \bullet a$ are homomorphisms of groups. 
The following lemma explains to what extent the biaction of $S$ on A depends on $\sigma$.

Lemma 2.11. Suppose $\sigma, \sigma^{\prime}: S \rightarrow \operatorname{Reg}_{E}(\mathbf{A})$ are maps, with $(x) \sigma,(x) \sigma^{\prime} \in(x) \Psi$, and let $\bullet$ and $\boldsymbol{A}$ be the corresponding biactions of $S$ on $\mathbf{A}$. Then

(i)

$$
x \boldsymbol{\bullet} a=x \bullet a \quad x \in S, a \in \mathbf{A}_{y},
$$

(ii) there exists a map $\beta: S \rightarrow A$, with $(x) \beta \in A_{x}$, such that

$$
\begin{gathered}
(x) \sigma^{\prime}=(x) \sigma((x) \beta) \eta, \\
a \bullet x=(a \bullet x)(y \bullet(x) \beta) \eta, \quad x \in S, a \in \mathbf{A}_{y},
\end{gathered}
$$

where $\eta: \mathbf{A}_{y x}\left(=\mathbf{A}_{(y x) * y x}\right) \rightarrow \operatorname{Reg}_{E}(\mathbf{A})$ is as in (2.6).

Proof. (i) is clear, since $x \mathbf{a} a=a \mathbf{A}\left(L\left(y^{*} y,(x y)^{*} x y\right)\right)=x \bullet a$.

(ii) Since $(x) \sigma,(x) \sigma^{\prime}$ belong to the same class $(x) \Psi$, by Proposition 2.5 , there must be elements $(x) \beta \in \mathbf{A}_{x}$ such that $(x) \sigma^{\prime}=(x) \sigma((x) \beta) \eta$. Let $(\alpha(x), \phi(x)): x x^{*} \rightarrow x^{*} x$ and $(\overline{\alpha(x)}, \overline{\phi(x)}): x x^{*} \rightarrow x^{*} x$ be unique representatives of $(x) \sigma$ and $(x) \sigma^{\prime}$ with domain $x x^{*}$ and range $x^{*} x$.

$$
\begin{aligned}
& \Longrightarrow[\overline{\alpha(x)}, \overline{\phi(x)}]=[\alpha(x), \phi(x)]\left[1_{x^{*} x}, \eta^{(x) \beta}\right] \\
& \Longrightarrow \overline{\phi_{e}}(x)=\left\{\phi(x)\left(\underline{C}(\alpha(x)) \eta^{(x) \beta}\right)\right\}_{e}=\phi_{e}(x) \eta_{x^{*} e x}^{(x) \beta},
\end{aligned}
$$

for every $e \in \omega\left(x x^{*}\right)$. Hence, for $h \in S\left(y^{*} y, x x^{*}\right)$,

$$
\begin{aligned}
a \mathbf{x}= & a \mathbf{A}\left(D\left(h, y^{*} y, x x^{*}\right)\right) \overline{\phi_{h x x^{*}}}(x) \mathbf{A}\left(L\left(x^{*} h x,(y x)^{*} y x\right)\right) \\
= & \left\{\left\{a \mathbf{A}\left(D\left(h, y^{*} y, x x^{*}\right)\right) \phi_{h x x^{*}}(x)\right\} \eta^{(x) \beta}{ }_{x^{*} h x}\right\} \mathbf{A}\left(L\left(x^{*} h x,(y x)^{*} y x\right)\right) \\
= & {\left[(x) \beta \mathbf{A}\left(L\left(x^{*} x, x^{*} h x\right)\right) \mathbf{A}\left(L\left(x^{*} h x,(y x)^{*} y x\right)\right)\right]^{-1} } \\
& \times\left[a \mathbf{A}\left(D\left(h, y^{*} y, x x^{*}\right)\right) \phi_{h x x^{*}}(x) \mathbf{A}\left(L\left(x^{*} h x,(y x)^{*} y x\right)\right)\right] \\
& \times\left[(x) \beta \mathbf{A}\left(L\left(x^{*} x, x^{*} h x\right)\right) \mathbf{A}\left(L\left(x^{*} h x,(y x)^{*} y x\right)\right)\right] \\
= & {\left[(x) \beta \mathbf{A}\left(L\left(x^{*} x,(y x)^{*} y x\right)\right)\right]^{-1}(a \bullet x)\left[(x) \beta \mathbf{A}\left(L\left(x^{*} x,(y x)^{*} y x\right)\right)\right] }
\end{aligned}
$$

by Lemma 2.10(i), since $(y x)^{*} y x \omega^{l} x^{*} h x \omega^{l} x^{*} x$

$$
\begin{aligned}
& =(y \bullet(x) \beta)^{-1}(a \bullet x)(y \bullet(x) \beta) \\
& =(a \bullet x)(y \bullet(x) \beta) \eta .
\end{aligned}
$$

Definition 2.12. Let $\Psi: S \rightarrow \operatorname{Reg}_{E}(\mathbf{A}) / \operatorname{Inn}_{E}(\mathbf{A})$ be an abstract kernel. Let $\sigma: S \rightarrow \operatorname{Reg}_{E}(\mathbf{A})$ and $p: S \times S \rightarrow \mathbf{A},(x, y) p \in \mathbf{A}_{x y}$, be maps such that

(i)

$$
(x) \sigma \in(x) \Psi
$$


2958 Idempotent-separating extensions of regular semigroups

(ii)

$$
(x) \sigma(y) \sigma=(x y) \sigma((x, y) p) \eta,
$$

(iii)

$$
(x y, z) p((x, y) p \bullet z)=(x, y z) p(x \bullet(y, z)) p
$$

where $\eta: \mathbf{A}_{x y}\left[=\mathbf{A}_{(x y) * x y}\right] \rightarrow \operatorname{Reg}_{E}(\mathbf{A})$ is as in (2.6) and the biaction $\bullet$ of $S$ on $\mathbf{A}$ is with respect to the map $\sigma$. Then the pair $(\sigma, p)$ is called a crossed pair.

Let $\Psi$ be an abstract kernel and let $\sigma, p$ be maps satisfying Definition 2.12(i) and (ii). In the next two lemmas, we establish some of the essential properties of the biaction $\bullet$ of $S$ on $\mathbf{A}$ induced by $\sigma$.

Lemma 2.13. Let $\Psi: S \rightarrow \operatorname{Reg}_{E}(\mathbf{A}) / \operatorname{Inn}_{E}(\mathbf{A})$ be an abstract kernel. Let $(\sigma, p)$ satisfy Definition 2.12(i) and (ii) and let $\bullet$ denote the biaction of $S$ on $\mathbf{A}$ induced by $\sigma$.

(i) If $(\alpha(e), \phi(e)): e \rightarrow e$ is a representative of $(e) \sigma$ with domain and range $e$, then $\phi_{e}(e)$ coincides with the inner automorphism defined by $(e, e) p$. More generally, if $e_{1} \omega^{r} e$, then for any $a \in \mathbf{A}_{e}=\mathbf{A}_{e}, a \bullet e_{1}=\left(e_{1}, e_{1}\right) p^{-1} a \mathbf{A}\left(e, c\left(e_{1} e, e_{1}\right)\right)\left(e_{1}, e_{1}\right) p$.

(ii) If $x \in S, e \in E(S)$, with $e x=x$, then for $a \in \mathbf{A}_{x}, e \bullet a=a$. If $(\sigma, p)$ also satisfies (2.48), then for $y \in S, e \in E(S),(e, e) p \bullet y=(e, y) p^{-1}(e, e y) p(e, y) p$.

Proof. (i) For $e \in E(S),(e) \sigma(e) \sigma=(e) \sigma((e, e) p) \eta \Rightarrow(e) \sigma=((e, e) p) \eta \Rightarrow \phi_{e}(e)=\eta_{e}(e, e) p$, the inner automorphism defined by the element $(e, e) p$.

If $e_{1} \omega^{r} e$, then $e_{1} e \in S\left(e, e_{1}\right)$ and for $a \in \mathbf{A}_{e}$, by (2.40),

$$
\begin{aligned}
a \bullet e_{1} & =a \mathbf{A}\left(D\left(e_{1} e, e, e_{1}\right)\right) \phi_{e 1}\left(e_{1}\right)=a \mathbf{A}\left(e, c\left(e_{1} e, e_{1}\right)\right) \eta_{e_{1}}{ }^{\left(e_{1}, e_{1}\right) p} \\
& =\left(e_{1}, e_{1}\right) p^{-1} a \mathbf{A}\left(e, c\left(e_{1} e, e_{1}\right)\right)\left(e_{1}, e_{1}\right) p .
\end{aligned}
$$

(ii) Clearly $e \cdot a=a \mathbf{A}\left(L\left(x^{*} x,(e x) * e x\right)\right)=a \mathbf{A}\left(L\left(x^{*} x, x^{*} x\right)\right)=a$. To prove the last assertion, let $y \in S, e \in E(S)$. Then, by (2.48), $(e, y) p((e, e) p \bullet y)=(e, e y) p(e \bullet(e, y)) p$ or $(e, e) p \bullet y=(e, y) p^{-1}(e, e y) p(e, y) p$, since $e(e y)=e y$ implies $(e \bullet(e, y)) p=(e, y) p$.

LEMma 2.14. Let $\Psi, \sigma, p$ be as in the first paragraph of Lemma 2.13. Then

(i)

$$
x \bullet(y \bullet a)=x y \bullet a, \quad x, y \in S, a \in \mathbf{A}_{z},
$$

(ii)

$$
x \bullet(b \bullet z)=(x \bullet b) \bullet z, \quad x, z \in S, b \in \mathbf{A}_{y},
$$

(iii)

$$
\begin{aligned}
(d \bullet y) \bullet z & =(d \bullet y z)(x \bullet(y, z) p) \eta \\
& =(x \bullet(y, z) p)^{-1}(d \bullet y z)(x \bullet(y, z) p) \quad y, z \in S, d \in \mathbf{A}_{x} .
\end{aligned}
$$


Proof. (i) is immediate from Lemma 2.10(i), since $(x y z)^{*} x y z \omega^{l}(y z)^{*} y z \omega^{l} z^{*} z$.

As before for $x \in S$, let $(\alpha(x), \phi(x)): x x^{*} \rightarrow x^{*} x$ denote the unique representative of $(x) \sigma$ with domain $x x^{*}$ and range $x^{*} x$. Let $x, y, z \in S$. Choose $h \in S\left(x^{*} x, y y^{*}\right), h_{2} \in S\left(y^{*} y\right.$, $\left.z z^{*}\right)$. Then $(x y)^{*} x y \mathbf{L} y^{*} h y$ and $y z(y z)^{*} \mathbf{R} y h_{2} y^{*}$. Let $h_{1} \in S\left((x y)^{*} x y, z z^{*}\right)=S\left(y^{*} h y, z z^{*}\right)$, $h_{3} \in S\left(x^{*} x, y z(y z)^{*}\right)=S\left(x^{*} x, y h_{2} y^{*}\right)$. Since $\phi(z): \mathbf{A}^{z z^{*}} \rightarrow \mathbf{A}^{z^{*} z} \underline{C}(\alpha(z))$ is a natural isomorphism, the diagram

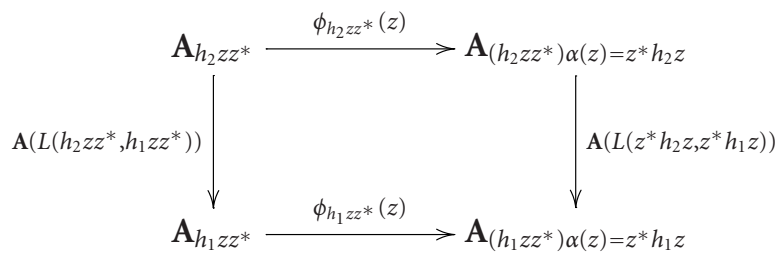

is commutative. Now, using Lemma 2.10(i) twice, we get

$$
\begin{aligned}
x \bullet(b \bullet z)= & b \mathbf{A}\left(D\left(h_{2}, y^{*} y, z z^{*}\right)\right) \phi_{h_{2} z z^{*}}(z) \mathbf{A}\left(L\left(z^{*} h_{2} z,(y z)^{*} y z\right)\right) \\
& \times \mathbf{A}\left(L\left((y z)^{*} y z,(x y z)^{*} x y z\right)\right) \\
= & b \mathbf{A}\left(D\left(h_{2}, y^{*} y, z z^{*}\right)\right) \phi_{h_{2} z z^{*}}(z) \mathbf{A}\left(L\left(z^{*} h_{2} z, z^{*} h_{1} z\right)\right) \mathbf{A}\left(L\left(z^{*} h_{1} z,(x y z)^{*} x y z\right)\right) \\
= & b \mathbf{A}\left[\left(D\left(h_{2}, y^{*} y, z z^{*}\right) L\left(h_{2} z z^{*}, h_{1} z z^{*}\right)\right)\right] \phi_{h_{1} z z^{*}}(z) \mathbf{A}\left(L\left(z^{*} h_{1} z,(x y z)^{*} x y z\right)\right) \\
= & b \mathbf{A}\left(D\left(h_{1}, y^{*} y, h_{1} z z^{*}\right)\right) \phi_{h_{1} z z^{*}}(z) \mathbf{A}\left(L\left(z^{*} h_{1} z,(x y z)^{*} x y z\right)\right) \\
& \quad \text { by Lemma } 2.10(\mathrm{iv}), \text { since } h_{2} \in S\left(y^{*} y, z z^{*}\right), h_{1} \omega^{l} y^{*} y, h_{1} \omega^{r} z z^{*} \\
= & b \mathbf{A}\left(L\left(y^{*} y,(x y)^{*} x y\right)\right) \mathbf{A}\left(D\left(h_{1},(x y)^{*} x y, z z^{*}\right)\right) \phi_{h_{1} z z^{*}}(z) \\
& \times \mathbf{A}\left(L\left(z^{*} h_{1} z,(x y z)^{*} x y z\right)\right) \\
& \quad \text { by Lemma } 2.10(\mathrm{ii}), \text { since }(x y)^{*} x y \omega^{l} y^{*} y \text { and } h_{1} \in S\left((x y)^{*} x y, z z^{*}\right) \\
= & (x \bullet b) \bullet z .
\end{aligned}
$$

Hence, the proof of (ii) is complete. To prove (iii), consider the diagram

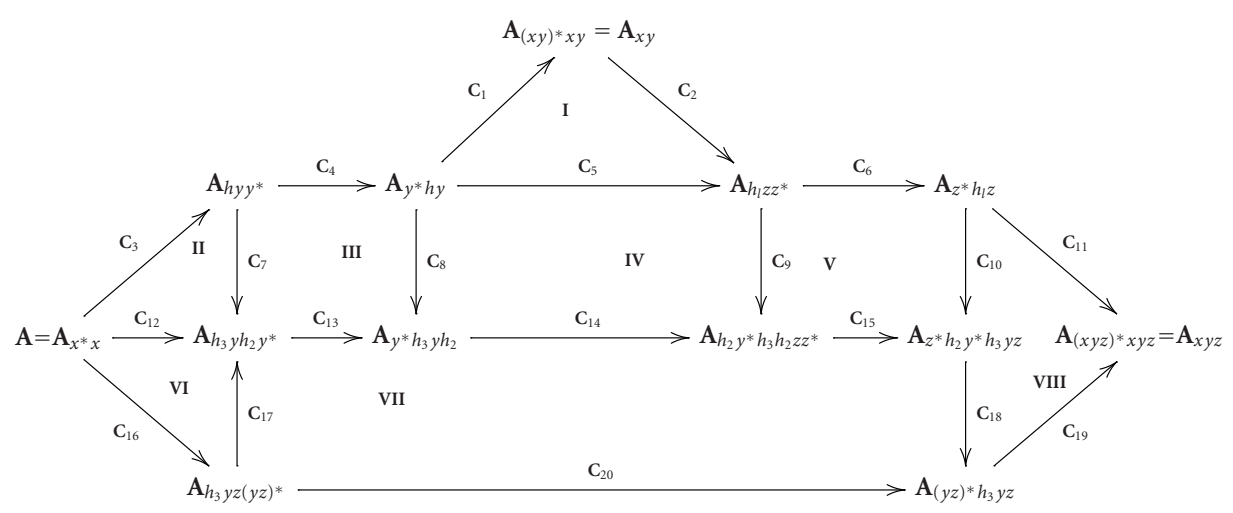


where

$$
\begin{aligned}
& C_{1}=\mathbf{A}\left(L\left(y^{*} h y,(x y)^{*} x y\right)\right), \quad C_{2}=\mathbf{A}\left(D\left(h_{1},(x y)^{*} x y, z z^{*}\right)\right), \\
& C_{3}=\mathbf{A}\left(D\left(h, x^{*} x, y y^{*}\right)\right), \quad C_{4}=\phi_{h y y^{*}}(y), \quad C_{5}=\mathbf{A}\left(D\left(h_{1}, y^{*} h y, z z^{*}\right)\right), \\
& C_{6}=\phi_{h_{1} z z^{*}}(h), \quad C_{7}=\mathbf{A}\left(D\left(h_{3} y y^{*}, h y y^{*}, y h_{2} y^{*}\right)\right), \\
& C_{8}=\mathbf{A}\left(D\left(y^{*} h_{3} y, y^{*} h y, y^{*} y h_{2}\right)\right), \quad C_{9}=\mathbf{A}\left(L\left(h_{1} z z^{*}, h_{2} y^{*} h_{3} y h_{2} z z^{*}\right)\right), \\
& C_{10}=\mathbf{A}\left(L\left(z^{*} h_{1} z, z^{*} h_{2} y^{*} h_{3} y z\right)\right), \quad C_{11}=\mathbf{A}\left(L\left(z^{*} h_{1} z,(x y z)^{*} x y z\right)\right), \\
& C_{12}=\mathbf{A}\left(D\left(h_{3}, x^{*} x, y h_{2} y^{*}\right)\right), \quad C_{13}=\phi_{h_{3} y h_{2} y^{*}}(y), \\
& C_{14}=\mathbf{A}\left(D\left(h_{2} y^{*} h_{3} y h_{2}, y^{*} h_{3} y h_{2}, z z^{*}\right)\right), \quad C_{15}=\phi_{h_{2} y^{*} h_{3} y h_{2} z z^{*}}(z), \\
& C_{16}=\mathbf{A}\left(D\left(h_{3}, x^{*} x, y z(y z)^{*}\right)\right), \quad C_{17}=\mathbf{A}\left(D\left(h_{3} y z(y z)^{*}, h_{3} y z(y z)^{*}, y h_{2} y^{*}\right)\right), \\
& C_{18}=\mathbf{A}\left(L\left(z^{*} h_{3} y^{*} h_{3} y z,(y z)^{*} h_{3} y z\right)\right), \quad C_{19}=\mathbf{A}\left(L\left((y z)^{*} h_{3} y z,(x y z)^{*} x y z\right)\right), \\
& C_{20}=\phi_{h_{3} y z(y z) *}(y z) \eta_{(y z) h_{3} y z}^{\left.(y, z) \mathbf{A}\left(L(y z)^{*} y z,(y z)^{*} h_{3} y z\right)\right)} \text {. }
\end{aligned}
$$

The commutativity of the diagram I follows from Lemma 2.10(ii), since $(x y)^{*} x y \mathbf{L} y^{*} h y$ and $h_{1} \in S\left((x y) * x y, z z^{*}\right)=S\left(y^{*} h y, z z^{*}\right)$. Since $y h_{2} y^{*} \omega y y^{*}$, by Lemma 2.10(iii), $h_{3} y y^{*} \in S\left(h y y^{*}, y h_{2} y^{*}\right)$ and the diagram II is commutative. The diagrams III and $\mathrm{V}$ are commutative, since $\phi(y)$ and $\phi(z)$ are natural isomorphisms. Next we show that the diagram IV is commutative. Now

$$
y^{*} h_{3} y h_{2} \omega^{l} h_{2} \Longrightarrow y^{*} h_{3} y h_{2} \mathbf{L} h_{2} y^{*} h_{3} y h_{2} \Longrightarrow h_{2} y^{*} h_{3} y h_{2} \in S\left(y^{*} h_{3} y h_{2}, z z^{*}\right) .
$$

Also

$$
h_{3} \omega^{r} y h_{2} y^{*} \Longrightarrow h_{1} z z^{*} h_{2} y^{*} h_{3}=h_{1}(x y)^{*} x h_{3} \Longrightarrow h_{1} z z^{*} \mathbf{L} h_{2} y^{*} h_{3} y z z^{*}
$$

since $z z^{*} h_{2}=h_{2}$ and $h_{3} y h_{1} z z^{*}=h_{3} x^{*} x y h_{1} z z^{*}=h_{3} y z z^{*}$. Take $e=y^{*} y, f=y^{*} h y, g=$ $h_{2}, h=y^{*} h_{3} y, k=h_{2} y^{*} h_{3} y h_{2}, m=h_{1}, n=z z^{*}$. The commutativity of the diagram IV now follows from Lemma 2.10(vi). Since $y z(y z)^{*} \mathbf{R} y h_{2} y^{*}$, the commutativity of the diagram VI follows from Lemma 2.10(v). Since $z^{*} h_{1} z \mathbf{L} z^{*} h_{2} y^{*} h_{3} y z \mathbf{L}(y z)^{*} h_{3} y z \mathbf{L}(x y z)^{*} x y z$, the diagram VIII is commutative by Lemma 2.10(i). Finally we establish the commutativity of the diagram VII. Put $c_{1}=c\left(y^{*} y h_{2}, h_{2}, h_{2} z z^{*}\right), c_{2}=c\left(y z(y z)^{*}, y h_{2} y^{*}\right), c_{3}=$ $c\left(z^{*} h_{2} z,(y z)^{*} y z\right),\left(\alpha_{1}, \phi_{1}\right)=y h_{2} y^{*} *(\alpha(y), \phi(y)),\left(\alpha_{2}, \phi_{2}\right)=(\alpha(z), \phi(z)) * z^{*} h_{2} z$. Then

$$
\begin{aligned}
{\left[(\alpha(y z), \phi(y z))\left(1_{(y z) * y z,} \eta^{(y, z) p}\right)\right] } \\
\quad=[\alpha(y z), \phi(y z)]\left[1_{(y z) * y z}, \eta^{(y, z) p}\right] \\
\quad=(y z) \sigma((y, z) p) \eta \\
\quad=(y) \sigma(z) \sigma \\
\quad=[\alpha(y), \phi(y)][\alpha(z), \phi(z)] \\
\quad=\left[\left((\alpha(y), \phi(y)) * y^{*} y h_{2}\right) \varepsilon\left(c_{1}\right)\left(h_{2} z z^{*} *(\alpha(z), \phi(z))\right)\right] \quad \text { by [10] } \\
\quad=\left[\left(\alpha_{1}, \phi_{1}\right) \varepsilon\left(c_{1}\right)\left(\alpha_{2}, \phi_{2}\right)\right] \quad \text { by }[13, \text { Proposition 3.2]. }
\end{aligned}
$$


Since $y z(y z)^{*} \mathbf{R} y h_{2} y^{*}$ and $z^{*} h_{2} z \mathbf{L}(y z)^{*} y z$, (1.9) implies that

$$
\begin{aligned}
(\alpha(y z), \phi(y z))\left(1_{(y z)^{*} y z}, \eta^{(y, z) p}\right) & \\
\quad= & \varepsilon\left(c_{2}\right)\left(\alpha_{1}, \phi_{1}\right) \varepsilon\left(c_{1}\right)\left(\alpha_{2}, \phi_{2}\right) \varepsilon\left(c_{3}\right) \\
& =\left(\alpha^{c_{2}}, \phi^{c_{2}}\right)\left(\alpha_{1}, \phi_{1}\right)\left(\alpha^{c_{1}}, \phi^{c_{1}}\right)\left(\alpha_{2}, \phi_{2}\right)\left(\alpha^{c_{3}}, \phi^{c_{3}}\right) .
\end{aligned}
$$

Therefore, the component at $e$ of the natural isomorphism defined by the left-hand side coincides with the component at $e$ of the natural isomorphism defined by the righthand side for each $e \in \omega\left(y z(y z)^{*}\right)$. In particular, by taking $e=h_{3} y z(y z)^{*}$ and noting that $\phi_{e}{ }^{c_{2}}=\mathbf{A}\left(D\left(e, e, y h_{2} y^{*}\right)\right)$ and $\phi^{c 1}{ }_{y^{*} h_{3} y h_{2}}=\mathbf{A}\left(D\left(h_{2} y^{*} h_{3} y h_{2}, y^{*} h_{3} y h_{2}, z z^{*}\right)\right)$, we obtain the commutativity of the diagram VII. As the interior diagrams are commutative, the outer diagram is commutative. Hence, for $d \in \mathbf{A}_{x}=\mathbf{A}_{x^{*} x}$,

$$
\begin{aligned}
(d \bullet y) \bullet z= & d \mathbf{A}\left(D\left(h, x^{*} x, y y^{*}\right)\right) \phi_{h y y^{*}}(y) \mathbf{A}\left(L\left(y^{*} h y,(x y)^{*} x y\right)\right) \\
& \times \mathbf{A}\left(D\left(h_{1},(x y)^{*} x y, z z^{*}\right)\right) \phi_{h_{1} z z^{*}}(z) \mathbf{A}\left(L\left(z^{*} h_{1} z,(x y z)^{*} x y z\right)\right) \\
= & d \mathbf{A}\left(D\left(h_{3}, x^{*} x, y z(y z)^{*}\right)\right) \phi_{e}(y z) \mathbf{A}\left(L\left((y z)^{*} h_{3} y z,(x y z)^{*} x y z\right)\right) \eta_{(x y z)^{*} x y z}^{(x \bullet y, z)} \\
= & (x \bullet(y, z) p)^{-1}(d \bullet y z)(x \bullet(y, z) p) .
\end{aligned}
$$

With these preliminaries we are now in a position to describe the extensions of $S$ by $\mathbf{A}$ which induce the given abstract kernel $\Psi$.

Theorem 2.15. Let $\Psi: S \rightarrow \operatorname{Reg}_{E}(\mathbf{A}) / \operatorname{Inn}_{E}(\mathbf{A})$ be an abstract kernel and let $(\sigma, p)$ be a crossed pair. Let

$$
T_{p}=\left\{(x, a): x \in S, a \in \mathbf{A}_{x}\right\} .
$$

Define a multiplication on $T_{p}$ by

$$
(x, a)(y, b)=(x y,(x, y) p(a \bullet y)(x \bullet b)) .
$$

Then $T_{p}$ is a regular semigroup with

$$
E\left(T_{p}\right)=\left\{\left(e,(e, e) p^{-1}\right): e \in E(S)\right\}
$$

The map $\pi_{p}: T_{p} \rightarrow S$ defined by $(x, a) \pi_{p}=x$, is an idempotent-separating homomorphism of $T_{p}$ onto $S$. For each $e \in E=E(S)$, define $\left(U_{p}\right)_{e}: \mathbf{A}_{e} \rightarrow \mathbf{A}_{e}{ }^{\pi p}$ by

$$
(a)\left(U_{p}\right)_{e}=\left(e,(e, e) p^{-1} a\right) \text {. }
$$

Then $U_{p}: e \rightarrow\left(U_{p}\right)_{e}$ defines a natural isomorphism between $\mathbf{A}$ and $\mathbf{A}^{\pi p}$. The triple $\left(T_{p}\right.$, $\left.\pi_{p} U_{p}\right)$ is an extension of $S$ by $\mathbf{A}$.

Proof. For $(x, a),(y, b),(z, c) \in T_{p}$, by (2.48), Lemma 2.14 , we easily prove that $((x, a)(y$, $b))(z, c)=(x, a)((y, b)(z, c))$. So the multiplication is associative. For each $e \in E(S)$, $\left(e,(e, e) p^{-1}\right)\left(e,(e, e) p^{-1}\right)=\left(e,(e, e) p\left((e, e) p^{-1} \bullet e\right)\left(e \bullet(e, e) p^{-1}\right)\right)=\left(e,(e, e) p^{-1}\right)$, since 
2962 Idempotent-separating extensions of regular semigroups

$(e, e) p^{-1} \bullet e=e \bullet(e, e) p^{-1}=(e, e) p^{-1}$, by Lemma 2.13(ii). Hence, $\left(e,(e, e) p^{-1}\right) \in E\left(T_{p}\right)$. On the other hand, $(e, a)(e, a)=(e, a) \Rightarrow(e e,(e, e) p(a \bullet e)(e \bullet a))=(e, a) \Rightarrow e e=e$ and $(e, e) p(a \bullet e)(e \bullet a)=a \Rightarrow e \in E(S)$ and $(e, e) p=a^{-1} \bullet e=(e, e) p^{-1} a^{-1}(e, e) p$ (by Lemma 2.13(i) and (ii)) $\Rightarrow e \in E(S)$ and $a=(e, e) p^{-1}$.

Hence, $E\left(T_{p}\right)=\left\{\left(e,(e, e) p^{-1}\right): e \in E(S)\right\}$. To prove $T_{p}$ is a regular semigroup, take any $(x, a) \in T_{p}$ and let $y$ be an inverse of $x$ in $S$. Put $b=y \bullet((x y, x y) p(x, y) p(a \bullet y))^{-1}$. Then $(y, b) \in T_{p}$, and $x \bullet b=x y \bullet((x y, x y) p(x, y) p(a \bullet y))^{-1}$ (by Lemma 2.14(i) $)=((x y$, $x y) p(x, y) p(a \bullet y))^{-1}$ (by Lemma 2.13(ii)). Then

$$
\begin{aligned}
(x, a)(y, b) & =(x y,(x, y) p(a \bullet y)(x \bullet b)) \\
& =\left(x y,(x, y) p(a \bullet y)(a \bullet y)^{-1}(x, y) p^{-1}(x y, x y) p^{-1}\right) \\
& =\left(x y,(x y, x y) p^{-1}\right) .
\end{aligned}
$$

Therefore, $(x, a)(y, b)(x, a)=\left(x y,(x y, x y) p^{-1}\right)(x, a)=\left(x y x,(x y, x) p\left((x y, x y) p^{-1} \bullet x\right)(x y \bullet\right.$ $a))=(x, a)$, since by Lemma 2.13(ii), $x y \bullet a=a$ and $(x y, x y) p \bullet x=(x y, x) p$ and $(y, b)(x$, $a)(y, b)=(y, b)\left(x y,(x y, x y) p^{-1}\right)=\left(y,(y, x y) p(b \bullet x y)\left(y \bullet(x y, x y) p^{-1}\right)\right)=(y, b)$, since

$$
\begin{aligned}
b \bullet x y & =\left[y \bullet\left(\left(a^{-1} \bullet y\right)(x, y) p^{-1}(x y, x y) p^{-1}\right)\right] \bullet x y \\
& =y \bullet\left[\left(\left(a^{-1} \bullet y\right)(x, y) p^{-1}(x y, x y) p^{-1}\right) \bullet x y\right] \quad \text { by Lemma 2.14(ii) } \\
& =y \bullet\left[\left(\left(a^{-1} \bullet y\right) \bullet x y\right)\left((x, y) p^{-1} \bullet x y\right)\left((x y, x y) p^{-1} \bullet x y\right)\right] \\
& =y \bullet\left(\left(x \bullet(y, x y) p^{-1}\right)\left(a^{-1} \bullet y\right)(x \bullet(y, x y) p)\left((x, y) p^{-1} \bullet x y\right)(x y, x y) p^{-1}\right)
\end{aligned}
$$

by Lemmas 2.14(iii) and 2.13(ii)

$=y \bullet\left(\left(x \bullet(y, x y) p^{-1}\right)\left(a^{-1} \bullet y\right)(x, y) p^{-1}\right) \quad$ using (2.48) for the triple $x, y, x y$

$=\left(y x \bullet(y, x y) p^{-1}\right)\left(y \bullet\left(a^{-1} \bullet y\right)(x, y) p^{-1}\right) \quad$ by Lemma 2.14(i)

$=(y, x y) p^{-1}\left(y \bullet\left(a^{-1} \bullet y\right)(x, y) p^{-1}\right)$ by Lemma 2.13(ii).

Hence, $(y, b)$ is an inverse of $(x, a)$, and $T_{p}$ is a regular semigroup. The map $\pi_{p}: T_{p} \rightarrow S$, $(x, a) \pi_{p}=x$, is clearly an idempotent-separating homomorphism from $T_{p}$ onto $S$ with $\mathbf{A}_{e}{ }^{\pi p}=\left\{(e, a): a \in \mathbf{A}_{e}\right\}$ for each $e \in E$. The map $U_{e}=\left(U_{p}\right)_{e}: \mathbf{A}_{e} \rightarrow \mathbf{A}_{e}{ }^{\pi p}$ defined by (2.65) is clearly a bijection. By Lemma 2.13, it is clear that $U_{e}$ is also a homomorphism.

We next show that the isomorphisms $U_{e}$ define a natural isomorphism $U_{p}: \mathbf{A} \rightarrow \mathbf{A}^{\pi p}$. We must show that for each morphism $\left(e, c\left(e_{0}, \ldots, e_{n}\right)\right): e \rightarrow f$ in $\underline{C}(E)$, the diagram

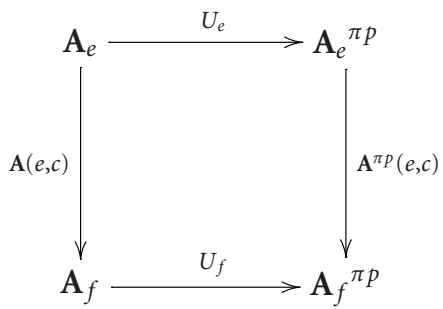


is commutative. Since $\left(e, c\left(e_{0}, \ldots, e_{n}\right)\right)=\left(e, e_{0}\right)\left(e_{0}, c\left(e_{0}, e_{1}\right)\right) \cdots\left(e_{n-1}, c\left(e_{n-1}, e_{n}\right)\right)$, it is enough to prove the commutativity of the diagram for morphisms of the form $(e, c(e, f))$, with $e \geq f$ or $e(\mathbf{R} \cup \mathbf{L}) f$.

Case $1(e \geq f)$. Let $a \in \mathbf{A}_{e}$. Then, since

$$
\begin{aligned}
\mathbf{A}^{\pi p}(e, f)= & \operatorname{Ker} \pi_{p}\left[\left(e,(e, e) p^{-1}\right),\left(f,(f, f) p^{-1}\right),\left(f,(f, f) p^{-1}\right)\right] \\
(a) U_{e} \mathbf{A}^{\pi p}(e, f)= & \left(e,(e, e) p^{-1} a\right) \mathbf{A}^{\pi p}(e, f) \\
= & \left(f,(f, f) p^{-1}\right)\left(e,(e, e) p^{-1} a\right)\left(f,(f, f) p^{-1}\right) \\
= & \left(e,(e, e) p^{-1} a\right)\left(f,(f, f) p^{-1}\right) \quad \text { by Definition } 1.1 \\
= & \left(f,(e, f) p\left(\left((e, e) p^{-1} a\right) \bullet f\right)\left(e \bullet(f, f) p^{-1}\right)\right) \\
= & \left(f,(e, f) p\left((e, e) p^{-1} \bullet f\right)(a \bullet f)(f, f) p^{-1}\right) \\
& \quad \text { since } e \bullet(f, f) p=(f, f) p \text { by Lemma 2.13(ii) } \\
= & \left(f,(a \bullet f)(f, f) p^{-1}\right) \quad \text { since }(e, e) p \bullet f=(e, f) p \text { by Lemma 2.13(ii) } \\
= & \left(f,(f, f) p^{-1}(a A(e, f))\right) \quad \text { by Lemma 2.13(i) } \\
= & a \mathbf{A}(e, f) U_{f} .
\end{aligned}
$$

Similarly we prove the diagram is commutative for other cases $e \mathbf{R} f$ and $e \mathbf{L} f$ also. Hence, by Definition 2.1, $\left(T_{p}, \pi_{p}, U_{p}\right)$ is an extension of $S$ by $\mathbf{A}$. The proof of Theorem 2.15 is complete.

We denote the extension $\left(T_{p}, \pi_{p}, U_{p}\right)$ by $(S, \sigma, p, \mathbf{A})$, and call the crossed extension of $S$ by $\mathbf{A}$ determined by the crossed pair $(\sigma, p)$.

Theorem 2.16. Let $\Psi: S \rightarrow \operatorname{Reg}_{E}(\mathbf{A}) / \operatorname{Inn}_{E}(\mathbf{A})$ be an abstract kernel and let $(\sigma, p)$ be a crossed pair, with $(x) \sigma \in(x) \Psi$ for every $x \in S$. Then the abstract kernel of the crossed extension $(S, \sigma, p, \mathbf{A})$ coincides with $\Psi$.

Proof. Define $j: S \rightarrow T_{p}$ by $(x) j=\left(x, 1_{x}\right)$, where $1_{x}$ denotes the identity element of $\mathbf{A}_{x}$. For each $(x, a) \in T_{p}$, let

$$
(x, a)^{*}=\left(x^{*}, x^{*} \bullet\left(\left(a^{-1} \bullet x^{*}\right)\left(x, x^{*}\right) p^{-1}\left(x x^{*}, x x^{*}\right) p^{-1}\right)\right) .
$$

Then the proof of Theorem 2.15 shows that $(x, a)^{*} \in V(x, a)$. Let $\bar{\mu}: T_{p} \rightarrow \operatorname{Reg}_{E}(\mathbf{A})$ be the idempotent-separating homomorphism defined by (2.8). Then

$$
(x) j \bar{\mu}=\left[\beta\left(x j,(x j)^{*}\right), \Psi\left(x j,(x j)^{*}\right)\right], \quad x \in S .
$$

The proof of the theorem follows once we show that the representative $\left(\beta\left(x j,(x j)^{*}\right)\right.$, $\left.\Psi\left(x j,(x j)^{*}\right)\right): x x^{*} \rightarrow x^{*} x$ of $(x) j \bar{\mu}$ and the representative $(\alpha(x), \phi(x)): x x^{*} \rightarrow x^{*} x$ of $(x) \sigma$ in $\mathbf{G}(\mathbf{A})$ are equal. From Remark 2.8(ii) and (2.13) it is clear that

$$
\beta\left(x j,(x j)^{*}\right)=\alpha(x)
$$

we next show that $\Psi\left(x j,(x j)^{*}\right)=\phi(x)$. To prove this we must show that $\Psi_{e}\left(x j,(x j)^{*}\right)=$ $\phi_{e}(x): \mathbf{A}_{\mathbf{e}} \rightarrow \mathbf{A}_{x^{*} e x}$ for each $e \in \omega\left(x x^{*}\right)$. For this purpose we first make some calculations. 
2964 Idempotent-separating extensions of regular semigroups

Let $e \in \omega\left(x x^{*}\right)$. Put $d=\left(x, x^{*}\right) p^{-1}\left(x x^{*}, x x^{*}\right) p^{-1}$. Then, by Lemmas 2.13(ii) and 2.14(i),

$$
\left(x^{*} \bullet d\right) \bullet e x=\left(x^{*} x \bullet\left(x^{*}, e x\right) p^{-1}\right)\left(x^{*} \bullet\left(x, x^{*} e x\right) p^{-1}\right) .
$$

Therefore, since $x^{*} x \bullet\left(x^{*}, e x\right) p^{-1}=\left(x^{*}, e x\right) p^{-1}$ by Lemma 2.13(ii),

$$
\left(x^{*} \cdot d\right) \bullet e x=\left(x^{*}, e x\right) p^{-1}\left(x^{*} \bullet\left(x, x^{*} e x\right) p^{-1}\right) .
$$

Putting $x=x^{*} e, y=x, z=x^{*}$ ex in (2.48),

$$
\left(x^{*} e x, x^{*} e x\right) p\left(x^{*} e, x\right) p \bullet x^{*} e x=\left(x^{*} e, e x\right) p\left(x^{*} e \bullet\left(x, x^{*} e x\right) p\right) .
$$

Since $\left(x^{*} e, x\right) p \cdot x^{*} e x=\left(x^{*} e x, x^{*} e x\right) p^{-1}\left(x^{*} e, x\right) p\left(x^{*} e x, x^{*} e x\right) p$ by Lemma 2.13(i) and $x^{*} e \bullet\left(x, x^{*}\right.$ ex $) p=\left(x^{*} \bullet\left(e \bullet\left(x, x^{*}\right.\right.\right.$ ex $\left.\left.) p\right)\right)=x^{*} \bullet\left(x, x^{*}\right.$ ex $) p$ by Lemma 2.13(ii), the above equation becomes

$$
\left(x^{*} e, x\right) p\left(x^{*} e x, x^{*} e x\right) p=\left(x^{*} e, e x\right) p\left(x^{*} \bullet\left(x, x^{*} e x\right) p\right)
$$

or

$$
\left(x^{*} \bullet\left(x, x^{*} e x\right) p^{-1}\right)=\left(x^{*} e x, x^{*} e x\right) p^{-1}\left(x^{*} e, x\right) p^{-1}\left(x^{*} e, e x\right) p .
$$

Since

$$
\begin{aligned}
\left(x, x^{*} e\right) p \bullet e & =(e, e) p^{-1}\left(x, x^{*} e\right) p(e, e) p \quad \text { by Lemma 2.13(i), } \\
(e, e) p\left(x, x^{*} e\right) p \bullet e & =\left(x, x^{*} e\right) p\left(x \bullet\left(x^{*} e, e\right) p\right) \quad \text { by }(2.48) \\
\Longrightarrow\left(x, x^{*} e\right) p(e, e) p & =\left(x, x^{*} e\right) p\left(x \bullet\left(x^{*} e, e\right) p\right) \Longrightarrow(e, e) p^{-1}=\left(x \bullet\left(x^{*} e, e\right) p^{-1}\right) \\
\Longrightarrow\left(x^{*} \bullet\left((e, e) p^{-1} \bullet x\right)\right) & =x^{*} x \bullet\left(\left(x^{*} e, e\right) p^{-1} \bullet x\right)=\left(x^{*} e, e\right) p^{-1} \bullet x .
\end{aligned}
$$

Also since

$$
\begin{aligned}
x^{*} e \bullet(e, x) p & =x^{*} \bullet(e \bullet(e, x) p)=x^{*} \bullet(e, x) p \quad \text { by Lemma 2.13(ii), } \\
\left(x^{*} e, x\right) p\left(x^{*} e, e\right) p \bullet x & =\left(x^{*} e, e x\right) p\left(x^{*} e \bullet(e, x) p\right) \quad \text { by }(2.48) \\
\Longrightarrow x^{*} \bullet(e, x) p & =\left(x^{*} e, e x\right) p^{-1}\left(x^{*} e, x\right) p\left(x^{*} e, e\right) p \bullet x \\
\Longrightarrow\left(x^{*} e, e\right) p \bullet x & =\left(x^{*} e, x\right) p^{-1}\left(x^{*} e, e x\right) p\left(x^{*} \bullet(e, x) p\right) .
\end{aligned}
$$

For any $a \in \mathbf{A}_{e}$, by (2.74), (2.77), (2.78), (2.79), and Lemma 2.13(i), it is easy to show

$$
(x j)^{*}\left(e,(e, e) p^{-1} a\right)(x j)=\left(x^{*} e x,\left(x^{*} e x, x^{*} e x\right) p^{-1}\right)\left(x^{*} \bullet(a \bullet x)\right) .
$$

But

$$
\begin{aligned}
x^{*} \bullet(a \bullet x) & =x^{*} \bullet\left(a \mathbf{A}\left(D\left(e, e, x x^{*}\right)\right) \phi_{e}(x) \mathbf{A}\left(L\left(x^{*} e x,(e x)^{*} e x\right)\right)\right) \\
& =x^{*} \bullet\left(a \phi_{e}(x) \mathbf{A}\left(L\left(x^{*} e x,(e x)^{*} e x\right)\right)\right) \quad \text { since } D\left(e, e, x x^{*}\right)=1_{e} \\
& =a \phi_{e}(x) \mathbf{A}\left(L\left(x^{*} e x,(e x)^{*} e x\right)\right) \mathbf{A}\left(L\left((e x)^{*} e x, x^{*} e x\right)\right) \quad \text { by }(2.39) \\
& =a \phi_{e}(x) \quad \text { by Lemma } 2.13(\mathrm{i}) .
\end{aligned}
$$


Hence, $(x j)^{*}\left(e,(e, e) p^{-1} a\right)(x j)=\left(x^{*} e x,\left(x^{*} e x, x^{*} e x\right) p^{-1}\right)(a) \phi_{e}(x)$. This implies $\Psi_{e}(x j$, $\left.(x j)^{*}\right)=\phi_{e}(x)$ for every $e \in \omega\left(x x^{*}\right)$, where we have identified $a \in \mathbf{A}_{e}$ with $\left(e,(e, e) p^{-1} a\right)$ under the isomorphism $\left(U_{p}\right)_{e}: \mathbf{A}_{e} \rightarrow \mathbf{A}_{e}{ }^{\pi P}$. Hence,

$$
\Psi\left(x j,(x j)^{*}\right)=\phi(x)
$$

The result now follows from (2.72) and (2.82).

Lemma 2.17 [9, Lemma 4.2]. Let $(T, \pi, 1)$ be an extension of $S$ by A. Let $j: S \rightarrow T$ be a map such that $j \pi=\mathbf{1}_{S}$ and let $\bullet$ denote the biaction of $S$ on $\mathbf{A}$ induced by the composite

$$
\sigma: S \stackrel{j}{\longrightarrow} T \stackrel{\bar{\mu}}{\longrightarrow} \operatorname{Reg}_{E}(\mathbf{A})
$$

where $\bar{\mu}$ is as in (2.16). Then $(x j) a(y j) b=(x j)(y j)(a \bullet y)(x \bullet b)$ for $x, y \in S, a \in \mathbf{A}_{\mathbf{x}}=$ $\mathbf{A}_{x^{*} x}, b \in \mathbf{A}_{\mathbf{y}}=\mathbf{A}_{y^{*} y}$.

Theorem 2.18. Let $\varepsilon_{T}=(T, \pi, \mathbf{1})$ be an extension of $S$ by $\mathbf{A}$ with abstract kernel $\Psi: S \rightarrow$ $\operatorname{Reg}_{E}(\mathbf{A}) / \operatorname{Inn}_{E}(\mathbf{A})$. Let $\sigma: S \rightarrow \operatorname{Reg}_{E}(\mathbf{A})$ be a map such that $(x) \sigma \in(x) \Psi$ for each $x \in S$. Then $\varepsilon_{T}$ is equivalent to a crossed extension of the form $(S, \sigma, p, \mathbf{A})$ with abstract kernel $\Psi$.

Proof. Let $\bar{\mu}: T \rightarrow \operatorname{Reg}_{E}(\mathbf{A})$ be the idempotent-separating homomorphism defined by (2.16). Using the commutativity of diagram (2.20), it is easy to see that every element in the class $(x) \Psi$ is of the form $\left[\beta\left(u, u^{\prime}\right), \Psi\left(u, u^{\prime}\right)\right]$ for some $u \in T, u^{\prime} \in V(u)$, with $u \pi=x$. So there is a map $j: S \rightarrow T$, with $j \pi=1_{S}$, such that $j \mu=\sigma$; in particular $(x) \sigma \in(x) \Psi$ for every $x \in S$. Since $((x j)(y j)) \pi=(x) j \pi(y) j \pi=x y=(x y) j \pi$, Lemma 1.6 defines a function $p: S \times S \rightarrow \mathbf{A},(x, y) p \in \mathbf{A}_{x y}$, such that $(x j)(y j)=(x y) j(x, y) p$. This implies, for $x, y \in S,(x) \sigma(y) \sigma=(x) j \bar{\mu}(y) j \bar{\mu}=((x j)(y j)) \bar{\mu}=(x y) j \bar{\mu}(x, y) p \bar{\mu}=(x y) \sigma(x, y) p \eta$, by (2.19). Again for $x, y, z \in S$, we have by Lemma 2.17, $(x j)((y j)(z j))=(x j)(y z) j(y, z) p=$ $(x j)(y z) j(x \bullet(y, z) p)=(x y z) j(x, y z) p(x \bullet(y, z) p)$, and $((x j)(y j))(z j)=(x y) j(x, y) p(z j)$ $=(x y) j(z j)((x, y) p \bullet z)=(x y z) j(x y, z) p((x, y) p \bullet z)$, where $\bullet$ denotes the biaction of $S$ on $\mathbf{A}$ induced by $\sigma$. Since the multiplication in $T$ is associative, by Lemma 1.6,

$$
(x y, z) p((x, y) p \bullet z)=(x, y z) p(x \bullet(y, z) p) .
$$

Thus $(\sigma, p)$ is a crossed pair.

Next we show that the extension $\varepsilon_{T}$ is equivalent to a crossed extension $(S, \sigma, p, \mathbf{A})$. Define $\theta: T_{p} \rightarrow T$ by $(x, a) \theta=(x j) a$. Then, by Lemma 2.17, $\theta$ is a homomorphism: $((x, a)(y$, b)) $\theta=(x y,(x, y) p(a \bullet y)(x \bullet b)) \theta=(x y) j(x, y) p(a \bullet y)(x \bullet b)=(x) j(y) j(a \bullet y)(x \bullet b)=$ $(x j) a(y j) b=(x, a) \theta(y, b) \theta$. From Lemma 1.6, we see that $\theta$ is a bijection and therefore an isomorphism. $\theta_{\pi}=\pi_{p}$, since $(x, a) \theta_{\pi}=((x j) a) \pi=x=(x, a) \pi_{p}$. Finally the diagram

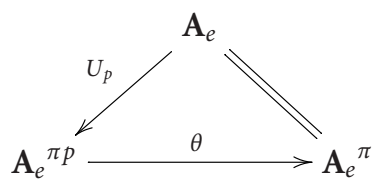


is commutative, since $(e j)(e j)=(e j)(e, e) p$ implies $e j=(e, e) p$ and hence for $a \in \mathbf{A}_{e}$, (a) $U_{p} \theta=\left(e,(e, e) p^{-1} a\right) \theta=(e j)(e, e) p^{-1} a=(e, e) p(e, e) p^{-1} a=a$. Hence, $\varepsilon_{T}$ is equivalent to a crossed extension $(S, \sigma, p, \mathbf{A})$. This completes the proof of Theorem 2.18.

Combining Theorems 2.15, 2.16, and 2.18, we obtain a complete description of extensions of $S$ by $A$ which induce the given abstract kernel $\Psi$ in terms of the crossed pairs $(\sigma, p)$.

\section{Obstructions to extensions}

Let $S^{I}$ be the regular semigroup obtained from $S$ by adjoining an identity element $I$ $(I \notin S)$. Extend the map $*: S \rightarrow S$ (see $(2.36))$ to $S^{I}$ by defining $I^{*}=I$. Now recall the category $D\left(S^{I}\right)$ [5] as follows. The objects are elements of $S^{I}$ and morphisms are the triples $\langle u, x, v\rangle: x \rightarrow y$ such that $u x v=y$. The morphism composition is defined by $\langle u, x, v\rangle\left\langle u^{\prime}\right.$, $\left.u x v, v^{\prime}\right\rangle=\left\langle u^{\prime} u, x, v v^{\prime}\right\rangle$. Let $\mathbf{F}: D\left(S^{I}\right) \rightarrow D\left(S^{I}\right)$ be the functor defined by $\mathbf{F}(x)=x^{*} x$ on objects of $D\left(S^{I}\right)$ and $\mathbf{F}\langle u, x, v\rangle=\left[x^{*} x, x^{*} x v y^{*} y, y^{*} u x\right]$ on morphisms $\langle u, x, v\rangle: x \rightarrow y$ of $D\left(S^{I}\right)$ [9]. A functor $\mathbf{G}: D\left(S^{I}\right) \rightarrow \mathbf{A b}$ is called a $D\left(S^{I}\right)$-module. For $D\left(S^{I}\right)$-modules $\mathbf{G}$ and $\mathbf{H}, D\left(S^{I}\right)$-homomorphism $\phi: \mathbf{G} \rightarrow \mathbf{H}$ is a natural transformation of functors. We denote by $\operatorname{hom}_{D\left(S^{I}\right)}(\mathbf{G}, \mathbf{H})$ the abelian group of all $D\left(S^{I}\right)$-homomorphisms from $\mathbf{G}$ to $\mathbf{H}$ and by $\operatorname{Mod}\left(D\left(S^{I}\right)\right)$ the category of $D\left(S^{I}\right)$-modules and $D\left(S^{I}\right)$-homomorphisms. $\operatorname{Mod}\left(D\left(S^{I}\right)\right)$ is an abelian category with enough injectives and projectives. Let $D\left(S^{I}\right)_{0}$ be the subcategory of $D\left(S^{I}\right)$ defined by the identity morphisms of $D\left(S^{I}\right)$. A $D\left(S^{I}\right)_{0}$-set is a functor $\Gamma: D\left(S^{I}\right)_{0} \rightarrow$ Sets from $D\left(S^{I}\right)_{0}$ to the category of sets, and $D\left(S^{I}\right)_{0}$-map is a natural transformation between two $D\left(S^{I}\right)_{0}$-sets. A $D\left(S^{I}\right)$-module (resp., $D\left(S^{I}\right)$-homomorphism) defines a $D\left(S^{I}\right)_{0}$-set (resp., $D\left(S^{I}\right)_{0}$-map) in an obvious manner. For more details, refer to [5].

If $\Gamma$ is a $D\left(S^{I}\right)_{0}$-set, then the free $D\left(S^{I}\right)$-module on $\Gamma$ is the $D\left(S^{I}\right)$-module $\mathbf{G}$ such that, for each object $y$ of $D\left(S^{I}\right), \mathbf{G}_{y}$ is the free abelian group generated by elements of the form $\left(a,\left\langle u, x, u^{\prime}\right\rangle\right), a \in \Gamma_{x}, x \in$ object $D\left(S^{I}\right), u x u^{\prime}=y$. If $\left\langle v, y, v^{\prime}\right\rangle: y \rightarrow z$ is a morphism of $D\left(S^{I}\right)$, then $\mathbf{G}\left\langle v, y, v^{\prime}\right\rangle: \mathbf{G}_{y} \rightarrow \mathbf{G}_{z}$ is defined by

$$
\left(a,\left\langle u, x, u^{\prime}\right\rangle\right) \mathbf{G}\left\langle v, y, v^{\prime}\right\rangle=\left(a,\left\langle v u, x, u^{\prime} v^{\prime}\right\rangle\right) .
$$

We identify $a \in \Gamma_{x}$ with $(a,\langle 1, x, 1\rangle)$ in $G_{x}$. For $n \geq 0$, let $x_{n}$ be the free $D\left(S^{I}\right)$-module on the $D\left(S^{I}\right)_{0}$-set $\Gamma_{n}$, where, for $n \geq 1$,

$$
\Gamma_{n}(x)=\left\{\left[u_{1}, \ldots, u_{n}\right] \in\left(S^{I}\right)^{n}: u_{1} u_{2} \cdots u_{n}=x\right\}
$$

and, for $n=0$,

$$
\Gamma_{0}(x)= \begin{cases}\{[1]\} & \text { if } x=1, \\ 0 & \text { if } x \neq 1, x \in S^{I} .\end{cases}
$$

Now we recall [5, Theorem 2.3]: the complex

$$
X \cdots \longrightarrow X_{n} \stackrel{\partial n}{\longrightarrow} X_{n-1} \stackrel{\partial_{n-1}}{\longrightarrow} \cdots \stackrel{\partial_{3}}{\longrightarrow} X_{2} \stackrel{\partial_{2}}{\longrightarrow} X_{1} \stackrel{\partial_{1}}{\longrightarrow} X_{0} \stackrel{\varepsilon}{\longrightarrow} \mathbf{Z}_{S^{I}} \longrightarrow 0
$$

is called the standard resolution of $\mathbf{Z}_{S^{I}}$. 
Let $\bar{\Gamma}_{n}(x)=\left\{\left[u_{1}, \ldots, u_{n}\right] \in \Gamma_{n}(x): u_{i} \neq 1, i=1,2, \ldots, n\right\}, n \geq 1$, and $\bar{\Gamma}_{n}=\cup \bar{\Gamma}_{n}(x), x \in$ $S^{I}$. Then the $D\left(S^{I}\right)_{0}$-set $\bar{\Gamma}_{n}$ freely generates a $D\left(S^{I}\right)$-submodule $\bar{X}_{n}$ of $X_{n}$. Put $\bar{X}_{0}=X_{0}$. Define $\partial_{n}$ as before, putting $\left[u_{1}, \ldots, u_{n}\right]=0$ whenever one of the $u_{i}$ is one. Then we obtain another projective resolution

$$
\bar{X} \cdots \longrightarrow \bar{X}_{n} \stackrel{\partial_{n}}{\longrightarrow} \bar{X}_{n-1} \stackrel{\partial_{n-1}}{\longrightarrow} \cdots \stackrel{\partial_{3}}{\longrightarrow} \bar{X}_{2} \stackrel{\partial_{2}}{\longrightarrow} \bar{X}_{1} \stackrel{\partial_{1}}{\longrightarrow} \bar{X}_{0} \stackrel{\varepsilon}{\longrightarrow} \mathbf{Z}_{S^{I}} \longrightarrow 0
$$

of $\mathbf{Z}_{S^{I}}$, called the normalised standard resolution of $\mathbf{Z}_{S^{I}}$.

Let $\mathbf{G} \in \operatorname{Mod}\left(\mathbf{D}\left(S^{I}\right)\right)$ and let

$$
\begin{gathered}
\operatorname{hom}_{D\left(S^{I}\right)}(\bar{X}, \mathbf{G}): 0 \longrightarrow \operatorname{hom}_{D\left(S^{I}\right)}\left(\bar{X}_{0}, \mathbf{G}\right) \stackrel{\partial_{1}{ }^{*}}{\longrightarrow} \operatorname{hom}_{D\left(S^{I}\right)}\left(\bar{X}_{1}, \mathbf{G}\right) \stackrel{\partial_{2}{ }^{*}}{\longrightarrow} \cdots \\
\stackrel{\partial_{n-1}{ }^{*}}{\longrightarrow} \operatorname{hom}_{D\left(S^{I}\right)}\left(\bar{X}_{n-1}, \mathbf{G}\right) \stackrel{\partial_{n}{ }^{*}}{\longrightarrow} \operatorname{hom}_{D\left(S^{I}\right)}\left(\bar{X}_{n}, \mathbf{G}\right) \stackrel{\partial_{n+1}{ }^{*}}{\longrightarrow} \cdots
\end{gathered}
$$

Definition 3.1. The $n$th cohomology group of $S^{I}$ with coefficients in $\mathbf{G}$, denoted by $\mathbf{H}^{n}\left(S^{I}\right.$, G), is defined by

$$
\mathbf{H}^{n}\left(S^{I}, \mathbf{G}\right)=\mathbf{H}^{n}\left[\operatorname{hom}_{D\left(S^{I}\right)}(\bar{X}, \mathbf{G})\right]=\operatorname{Ker} \partial_{n+1}{ }^{*} / \operatorname{Im} \partial_{n}{ }^{*} .
$$

The elements of $\operatorname{hom}_{D\left(S^{I}\right)}(\bar{X}, \mathbf{G})$ are called (normalized) $n$-cochains. The elements of $\operatorname{Ker} \partial_{n+1}{ }^{*}$ are called (normalized) $n$-cocycles and the elements of $\operatorname{Im} \partial_{n}{ }^{*}$ are called (normalized) $n$-coboundaries. Two $n$-cocycles $k_{1}, k_{2} \in \operatorname{Ker} \partial_{n+1}{ }^{*}$ are called cohomologous if they differ by a coboundary.

Let $\mathbf{A}: \underline{C}(E) \rightarrow \mathbf{G R}$ be a group $E$-diagram that factors through $\mathbf{D}(B(E))$ and let $\mathbf{Z}(\mathbf{A})$ be the centre of $\mathbf{A}$. For each $x \in S$, let $\overline{\mathbf{Z}(\mathbf{A})_{x}}=\mathbf{Z}(\mathbf{A})_{x^{*} x}$ and let

$$
\overline{\mathbf{Z}(\mathbf{A})}=\bigcup_{x \in S} \overline{\mathbf{Z}(\mathbf{A})_{x}}
$$

be the disjoint union of $\overline{\mathbf{Z}(\mathbf{A})_{x}}$ 's. Remark that $\overline{\mathbf{Z}(\mathbf{A})_{x}}$ is contained in the centre of $\overline{\mathbf{A}}_{x}$, where as in the previous section $\overline{\mathbf{A}}=\bigcup_{x \in S} \overline{\mathbf{A}}_{x}$ with $\mathbf{A}_{x}=\overline{\mathbf{A}}_{x^{*} x}$.

Suppose $\Psi: S \rightarrow \operatorname{Reg}_{E}(\mathbf{A}) / \operatorname{Inn}_{E}(\mathbf{A})$ is an abstract kernel. Then the composite $S \stackrel{\Psi}{\longrightarrow}$ $\left(\operatorname{Reg}_{E}(\mathbf{A}) / \operatorname{Inn}_{E}(\mathbf{A})\right) \stackrel{V}{\longrightarrow} \operatorname{Reg}_{E}(\mathbf{Z}(\mathbf{A}))$ is an idempotent-separating homomorphism. Since A and hence $\mathbf{Z}(\mathbf{A})(: \underline{C}(E) \rightarrow \mathbf{A b})$ factors through $D(B(E))$, by Remark 2.9, $\Psi v$ induces a functor $\check{\mathbf{Z}}(\mathbf{A})=\check{\mathbf{Z}}(\mathbf{A})_{\Psi v}: \mathbf{D}(S) \rightarrow \mathbf{A b}$. Let $\check{\mathbf{Z}}(\mathbf{A})^{0}: \mathbf{D}\left(S^{I}\right) \rightarrow$ Ab be the extension of $\check{\mathbf{Z}}(\mathbf{A})$ such that $\check{\mathbf{Z}}(\mathbf{A})_{I}{ }^{0}=\{0\}$ and let $\check{\mathbf{Z}}(\mathbf{A})^{0} \mathbf{F}$ be the composite $D\left(S^{I}\right) \stackrel{\mathbf{F}}{\longrightarrow} \mathbf{D}\left(S^{I}\right) \stackrel{\check{\mathbf{Z}}(\mathbf{A})^{0}}{\longrightarrow} \mathbf{A b}$. In this section, we associate with the abstract kernel $\Psi$ a 3-dimensional cohomology class $[k] \in \mathbf{H}^{3}\left(S^{I}, \check{\mathbf{Z}}(\mathbf{A})^{0} \mathbf{F}\right)$ and show that $\Psi$ admits an extension if and only if $[k]=0$. We also show that if $\Psi$ has an extension, then the set of all equivalence classes of extensions of $S$ by $A$ is in bijective correspondence with the set $\mathbf{H}^{2}\left(S^{I}, \check{\mathbf{Z}}(\mathbf{A})^{0} \mathbf{F}\right)$. 
Let $\sigma: S \rightarrow \operatorname{Reg}_{E}($ A) be any map such that $(x) \sigma \in(x) \Psi$. As before for each $x \in S$, let $(\alpha(x), \phi(x)): x x^{*} \rightarrow x^{*} x$ denote the unique representative of $(x) \sigma$ in $\mathbf{G}(\mathbf{A})$ with domain $x x^{*}$ and range $x^{*} x$ and let $(\alpha(x), \overline{\phi(x)}): x x^{*} \rightarrow x^{*} x$ denote the element of $\mathbf{G}(\mathbf{Z}(\mathbf{A}))$ determined by $(\alpha(x), \phi(x))$ (see (2.13)) so that $(x) \Psi v=(x) \sigma u=[\alpha(x), \overline{\phi(x)}]$. The biaction of $S$ on A defined by $\sigma$ induces by restriction a biaction of $S$ on $\overline{\mathbf{Z}(\mathbf{A})}$ which coincides with the one induced by the composite $\Psi v=\sigma u: S \rightarrow \operatorname{Reg}_{E}(\mathbf{A}) \rightarrow \operatorname{Reg}_{E}(\mathbf{Z}(\mathbf{A}))$. In particular, the induced biaction of $S$ on $\overline{\mathbf{Z}(\mathbf{A})}$ is independent of the chosen $\sigma$. We next see the relation between this biaction and the functor $\check{\mathbf{Z}}(\mathbf{A})^{0} \mathbf{F}: D\left(S^{I}\right) \rightarrow \mathbf{A b}$. Let $x \in S$, $a \in \overline{\mathbf{Z}(\mathbf{A})}_{y}=\mathbf{Z}(\mathbf{A})_{y^{*} y}=\left(\check{\mathbf{Z}}(\mathbf{A})^{0} \mathbf{F}\right)_{y}$. Then by (2.23) and (2.39) we have

$$
\begin{aligned}
a\left(\check{\mathbf{Z}}(\mathbf{A})^{0} \mathbf{F}\langle x, y, I\rangle\right)= & a \check{\mathbf{Z}}(\mathbf{A})^{0}\left[y^{*} y, y^{*} y(x y)^{*} x y,(x y)^{*} x y\right] \\
= & a \check{\mathbf{Z}}(\mathbf{A})\left[y^{*} y, y^{*} y(x y)^{*} x y,(x y)^{*} x y\right] \\
= & a \mathbf{Z}(\mathbf{A})\left(L\left(y^{*} y,(x y)^{*} x y\right)\right)=x \bullet a, \\
a\left(\check{\mathbf{Z}}(\mathbf{A})^{0} F\langle I, y, x\rangle\right)= & a \check{\mathbf{Z}}(\mathbf{A})^{0}\left[y^{*} y, y^{*} y x,(y x)^{*} y\right] \\
= & a \check{\mathbf{Z}}(\mathbf{A})\left[y^{*} y, y^{*} y x,(y x)^{*} y\right] \\
= & a \check{\mathbf{Z}}(\mathbf{A})\left[y^{*} y, y^{*} y x,(y x)^{*} y h\right] \quad \text { by }(1.2) \\
= & a \check{\mathbf{Z}}(\mathbf{A})\left\{\left[y^{*} y, y^{*} y x x^{*}, h\right]\right. \\
& \left.\times\left[h x x^{*}, h x, x^{*} h x x^{*}\right]\left[x^{*} h x, x^{*} h x,(y x)^{*} y x\right]\right\} \\
= & a \mathbf{Z}(\mathbf{A})\left(D\left(h, y^{*} y, x x^{*}\right)\right) \check{\mathbf{Z}}(\mathbf{A})\left[h x x^{*}, h x, x^{*} h x x^{*}\right] \\
& \times \mathbf{Z}(\mathbf{A})\left(L\left(x^{*} h x,(y x)^{*} y x\right)\right) \quad \text { by }(2.23) \\
= & a \mathbf{Z}(\mathbf{A})\left(D\left(h, y^{*} y, x x^{*}\right)\right) \bar{\phi}_{h x x^{*}}(h x) \mathbf{Z}(\mathbf{A})\left(L\left(x^{*} h x,(y x)^{*} y x\right)\right)
\end{aligned}
$$

by Remark 2.9

$$
\begin{aligned}
& =a \mathbf{Z}(\mathbf{A})\left(D\left(h, y^{*} y, x x^{*}\right)\right) \bar{\phi}_{h x x^{*}}(x) \mathbf{Z}(\mathbf{A})\left(L\left(x^{*} h x,(y x)^{*} y x\right)\right) \\
& =a \cdot x \text { by }(2.40),
\end{aligned}
$$

where $h \in S\left(y^{*} y, x x^{*}\right)$, and the components $\bar{\phi}_{h x x^{*}}(h x)$ of $\bar{\phi}(h x)$ and $\bar{\phi}_{h x x^{*}}(x)$ of $\bar{\phi}(x)$ are equal since $[\alpha(h x), \bar{\phi}(h x)]=(h x) \Psi v=\left(h x x^{*}\right) \Psi v(x) \Psi v=\left[1_{h x x^{*}}, \mathbf{1}_{h x x^{*}}\right][\alpha(x), \bar{\phi}(x)]=$ $\left[h x x^{*} *(\alpha(x), \phi(x))\right]$. Thus we have $x \bullet a=a\left(\check{\mathbf{Z}}(\mathbf{A})^{0} \mathbf{F}\langle x, y, I\rangle\right)$ and $a \bullet x=a\left(\check{\mathbf{Z}}(\mathbf{A})^{0} \mathbf{F}\langle I, y\right.$, $x\rangle$.

Next we describe the cohomology groups. Consider the normalized standard resolution (3.5). Since the $D\left(S^{I}\right)$-module $\bar{X}_{n}$ 's are free on $\bar{\Gamma}_{n}$ 's and since $\left(\check{\mathbf{Z}}(\mathbf{A})^{0} \mathbf{F}\right)_{I}=\{0\}$, we have

$$
\begin{aligned}
\operatorname{hom}_{D\left(S^{I}\right)}\left(\bar{X}_{n}, \check{\mathbf{Z}}(\mathbf{A})^{0} \mathbf{F}\right) & =\operatorname{hom}_{D\left(S^{I}\right)_{0}}\left(\bar{\Gamma}_{n}, \check{\mathbf{Z}}(\mathbf{A})^{0} \mathbf{F}\right) \\
& =\left\{\alpha: \underset{(n \text { times })}{S \times S} \times \cdots \times S \longrightarrow \overline{\mathbf{Z}(\mathbf{A})}:\left(x_{1}, x_{2}, \ldots, x_{n}\right) \in \overline{\mathbf{Z}(\mathbf{A})_{x_{1} x_{2}} \cdots x_{n}}\right\} .
\end{aligned}
$$


Hence, we may regard an $n$-cochain as a map $\alpha: \underset{(n \text { times })}{S \times S} \times \cdots \times S \rightarrow \overline{\mathbf{Z}(\mathbf{A})}$, with $\left(x_{1}, x_{2}\right.$, $\left.\ldots, x_{n}\right) \in \overline{\mathbf{Z}(\mathbf{A})_{x_{1} x_{2}} \ldots x_{n}}$. The coboundary $\partial_{n}{ }^{*} \alpha$ of an $n-1$ cochain $\alpha$ is given by the formula

$$
\begin{aligned}
\left(x_{1}, x_{2}, \ldots, x_{n}\right) \partial_{n}{ }^{*} \alpha= & \left(x_{2}, x_{3}, \ldots, x_{n}\right) \alpha \check{\mathbf{Z}}(\mathbf{A})^{0} \mathbf{F}\left\langle x_{1}, x_{2}, \ldots, x_{n}, I\right\rangle \\
& +\sum_{i=1}^{n-1}(-1)^{i}\left(x_{1}, x_{2}, \ldots, x_{i} x_{i+1}, \ldots, x_{n}\right) \alpha \\
& +(-1)^{n}\left(x_{1}, x_{2}, \ldots x_{n-1}\right) \alpha \check{\mathbf{Z}}(\mathbf{A})^{0} \mathbf{F}\left\langle I, x_{1}, x_{2}, \ldots, x_{n-1}, x_{n}\right\rangle \\
= & x_{1} \bullet\left(x_{2}, x_{3}, \ldots, x_{n}\right) \alpha+\sum_{i=1}^{n-1}(-1)^{i}\left(x_{1}, x_{2}, \ldots, x_{i} x_{i+1}, \ldots, x_{n}\right) \alpha \\
& +(-1)^{n}\left(x_{1}, x_{2}, \ldots, x_{n-1}\right) \alpha \bullet x_{n} .
\end{aligned}
$$

From now on we write the group operation as multiplication. Note that a 2-cochain $\alpha$ : $S \times S \rightarrow \overline{\mathbf{Z}(\mathbf{A})},(x, y) \in \overline{\mathbf{Z}(\mathbf{A})_{x y}}$, is a 2-cocycle if

$$
(x y, z) \alpha((x, y) \alpha \bullet z)=(x, y z) \alpha(x \bullet(y, z) \alpha)
$$

for all $x, y, z \in S$; $\alpha$ is a coboundary if and only if there exists a 1 -cochain $\beta: S \rightarrow \overline{\mathbf{Z}(\mathbf{A})}$, $(x) \beta \in \overline{\mathbf{Z}(\mathbf{A})})_{x}$, such that

$$
(x, y) \alpha=(x \bullet(y) \beta)(x y) \beta^{-1}((x) \beta \bullet y)
$$

for all $x, y \in S$. Similarly a 3-cocycle $k$ is a map $k: S \times S \times S \rightarrow \overline{\mathbf{Z}(\mathbf{A})},(x, y, z) k \in \overline{\mathbf{Z}(\mathbf{A})}_{x y z}$, such that

$$
(x y, z, t) k(x, y, z t) k=((x, y, z) k \bullet t)(x, y z, t) k(x \bullet(y, z, t) k)
$$

for all $x, y, z, t \in S$; $k$ is a coboundary if and only if there exists a 2-cochain $\alpha: S \times S \rightarrow$ $\overline{\mathbf{Z}(\mathbf{A})},(x, y) \in \overline{\mathbf{Z}(\mathbf{A})}_{x y}$, such that

$$
(x, y, z) k=(x \bullet(y, z) \alpha)(x y, z) \alpha^{-1}(x, y z) \alpha((x, y) \alpha \bullet z)^{-1}
$$

for all $x, y, z \in S$. For $n=2,3$, let $\mathbf{Z}^{n}\left(\mathbf{S}^{I}, \check{\mathbf{Z}}(\mathbf{A})^{0} \mathbf{F}\right)$ denote the abelian group of all $n$-cocycles and let $\mathbf{B}^{n}\left(\mathbf{S}^{I}, \check{\mathbf{Z}}(\mathbf{A})^{0} \mathbf{F}\right) \subseteq \mathbf{Z}^{n}\left(\mathbf{S}^{I}, \check{\mathbf{Z}}(\mathbf{A})^{0} \mathbf{F}\right)$ be the subgroup of all coboundaries. Then

$$
\mathbf{H}^{n}\left(S^{I}, \check{\mathbf{Z}}(\mathbf{A})^{0} \mathbf{F}\right)=\frac{\mathbf{Z}^{n}\left(\mathbf{S}^{I}, \check{\mathbf{Z}}(\mathbf{A})^{0} \mathbf{F}\right)}{\mathbf{B}^{n}\left(\mathbf{S}^{I}, \check{\mathbf{Z}}(\mathbf{A})^{0} \mathbf{F}\right)}
$$

Now we proceed to show that the abstract kernel $\Psi: S \rightarrow \operatorname{Reg}_{E}(\mathbf{A}) / \operatorname{Inn}_{E}(\mathbf{A})$ defines an element in the cohomology group $\mathbf{H}^{3}\left(\mathbf{S}^{I}, \check{\mathbf{Z}}(\mathbf{A})^{0} \mathbf{F}\right)$, the vanishing of which is necessary and sufficient for the existence of extensions of $\Psi$. 
We fix a map $\sigma: S \rightarrow \operatorname{Reg}_{E}(\mathbf{A})$ such that $(x) \sigma \in(x) \Psi$ for all $x \in S$. Let $\bullet$ denote the biaction of $S$ on $\mathbf{A}$ induced by $\sigma$. As before we denote by $(\alpha(x), \phi(x)): x x^{*} \rightarrow x^{*} x$ the unique representative of $(x) \sigma$ in $\mathbf{G}(\mathbf{A})$ with domain $x x^{*}$ and range $x^{*} x$. Since $(x \sigma)(y \sigma)$ and $(x y) \sigma$ both belong to the same class $(x y) \Psi$, we can choose a function $p: S \times S \rightarrow \mathbf{A}$, $(x, y) p \in \mathbf{A}_{x y}$, such that

$$
(x) \sigma(y) \sigma=(x y) \sigma((x, y) p) \eta,
$$

where $\eta: \mathbf{A}_{x y},=\mathbf{A}_{(x y) * x y} \rightarrow \operatorname{Reg}_{E}(\mathbf{A})$ is as before.

Before proceeding further, let us first prove the following.

Lemma 3.2. For $a \in \mathbf{A}_{x}, b \in \mathbf{A}_{y}$,

$$
(x \sigma)(a) \eta(y \sigma)(b) \eta=(x y) \sigma((x, y) p(a \bullet y)(x \bullet b)) \eta .
$$

Proof. Consider the diagram

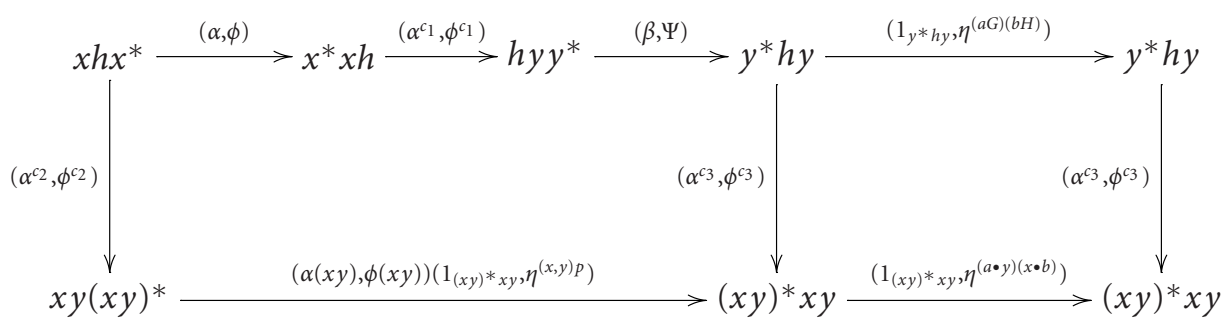

where

$$
\begin{gathered}
h \in S\left(x^{*} x, y y^{*}\right), \quad c_{1}=c\left(x^{*} x h, h, h y y^{*}\right), \quad c_{2}=c\left(x h x^{*}, x y(x y)^{*}\right), \\
c_{3}=c\left(y^{*} h y,(x y)^{*} x y\right), \\
\varepsilon\left(c_{1}\right)=\left(\alpha^{c_{1}}, \phi^{c_{1}}\right), \quad \varepsilon\left(c_{2}\right)=\left(\alpha^{c_{2}}, \phi^{c_{2}}\right), \quad \varepsilon^{2}\left(c_{3}\right)=\left(\alpha^{c_{3}}, \phi^{c_{3}}\right), \\
(\alpha, \phi)=(\alpha(x), \phi(x)) * x^{*} x h: x h x^{*} \longrightarrow x^{*} x h, \\
(\beta, \Psi)=h y y^{*} *\left(\alpha(y), \phi(y): h y y^{*}\right) \longrightarrow y^{*} h y, \\
H=\mathbf{A}\left(L\left(y^{*} y, y^{*} h y\right)\right), \\
G=\mathbf{A}\left(L\left(x^{*} x, x^{*} x h\right)\right)\left(\phi^{c_{1}}\right)_{x^{*} x h} \Psi_{h y y^{*}} \\
=\mathbf{A}\left(L\left(x^{*} x, x^{*} x h\right)\right) \mathbf{A}\left(D\left(h, x^{*} x h, y y^{*}\right)\right) \Psi_{h y y^{*}} \\
=\mathbf{A}\left(D\left(h, x^{*} x, y y^{*}\right)\right) \Psi_{h y y^{*}} \quad \text { by Lemma } 2.10(\mathrm{ii}), \\
a \bullet y=(a) G \mathbf{A}\left(L\left(y^{*} h y,(x y)^{*} x y\right)\right)=(a) G\left(\phi^{c_{3}}\right)_{y^{*} h y} \text { by }[9,(1.8)], \\
x \bullet b=(b) \mathbf{A}\left(L\left(y^{*} y,(x y)^{*} x y\right)\right)=(b) \mathbf{A}\left(L\left(y^{*} h y,(x y)^{*} x y\right)\right)=(b) H\left(\phi^{c_{3}}\right)_{y^{*} h y} \\
\text { by }[10,(1.8)] .
\end{gathered}
$$


Since $(x \sigma)(y \sigma)=(x y) \sigma((x, y) p) \eta$, the first rectangle is commutative. The second rectangle is also commutative, since for $d \in \mathbf{A}_{y^{*} h y}$,

$$
\begin{aligned}
d\left(\eta^{(a G)(b H)}\right)_{y^{*} h y}\left(\phi^{c_{3}}\right)_{y^{*} h y} \\
\quad=\left(((a G)(b H))^{-1} d(a G)(b H)\right)\left(\phi^{c_{3}}\right)_{y^{*} h y} \\
\quad=((a G)(b H))^{-1}\left(\phi^{c_{3}}\right)_{y^{*} h y} d\left(\phi^{c_{3}}\right)_{y^{*} h y}((a G)(b H))\left(\phi^{c_{3}}\right)_{y^{*} h y} \\
=((a \bullet y)(x \bullet b))^{-1}\left(d \phi^{c_{3}}\right)_{y^{*} h y}((a \bullet y)(x \bullet b)) \\
=(d)\left(\phi^{c_{3}}\right)_{y^{*} h y}\left(\eta^{(a \bullet y)(x \bullet b)}\right)(x y)^{*} x y \\
=(d)\left(\phi^{c_{3}}\left(\underline{C}\left(\alpha^{c_{3}}\right) \eta^{(a \bullet y)(x \bullet b)}\right)\right)_{y^{*} h y} .
\end{aligned}
$$

Hence, the outer diagram is commutative. Now

$$
\begin{aligned}
(x \sigma)(a) \eta(y) \sigma(b) \eta & =[\alpha(x), \phi(x)]\left[1_{x^{*} x}, \eta^{a}\right][\alpha(y), \phi(y)]\left[1_{y^{*} y}, \eta^{b}\right] \\
& =\left[(\alpha, \phi)\left(\left(1_{x^{*} x}, \eta^{a}\right) * x^{*} x h\right) \varepsilon\left(c_{1}\right)(\beta, \Psi)\left(y^{*} h y^{*}\left(1_{y^{*} y}, \eta^{b}\right)\right)\right] \quad \text { by }(1.10) \\
& =\left[(\alpha, \phi) \varepsilon\left(c_{1}\right)(\beta, \Psi)\left(1_{y^{*} h y}, \eta^{a G}\right)\left(1_{y^{*} h y}, \eta^{b H}\right)\right] \\
& =\left[(\alpha(x y), \phi(x y))\left(1_{(x y) * x y}, \eta^{(x, y) p(a \bullet y)(x \bullet b)}\right)\right] \quad \text { by the diagram } \\
& =[\alpha(x y), \phi(x y)]\left[1_{(x y) * x y}, \eta^{(x, y) p(a \bullet y)(x \bullet b)}\right] \\
& =(x y) \sigma((x, y) p(a \bullet y)(x \bullet b)) \eta .
\end{aligned}
$$

Hence, the proof of the lemma is complete.

Let $\sigma$ and $p$ be as before. Using (3.17) and Lemma 3.2, we get

$$
\begin{aligned}
& ((x \sigma)(y \sigma))(z \sigma)=(x y) \sigma((x, y) p) \eta(z \sigma)=(x y z) \sigma((x y, z) p((x, y) p \bullet z)) \eta, \\
& (x \sigma)((y \sigma)(z \sigma))=(x \sigma)(y z) \sigma((y, z) p) \eta=(x y z) \sigma((x, y z) p(x \bullet(y, z) p)) \eta .
\end{aligned}
$$

Since the multiplication in $\operatorname{Reg}_{E}(\mathbf{A})$ is associative, by Lemma 1.6,

$$
((x y, z) p((x, y) p \bullet z)) \eta=((x, y z) p(x \bullet(y, z) p)) \eta .
$$

The exactness of the sequence in Proposition 2.5 gives us a 3-cochain $k: S \times S \times S \rightarrow \overline{\mathbf{Z}(\mathbf{A})}$ such that

$$
(x y, z) p((x, y) p \bullet z)=(x, y z) p(x \bullet(y, z) p)(x, y, z) k
$$

for all $x, y, z \in S$.

Lemma 3.3. The map $k: S \times S \times S \rightarrow \overline{\mathbf{Z}(\mathbf{A})}$ is a 3-cocycle.

Proof. We must show that $k$ satisfies (3.14). Let $x, y, z, t \in S$. Following [11], it is easy to calculate the expression

$$
L=(x y z, t) p[(x y, z) p((x, y) p \bullet z)] \bullet t
$$


in two ways. In the first way using (3.27) and Lemma 2.14, we easily get

$$
L=(x, y z t) p(x \bullet(y, z t) p)(x y \bullet(z, t) p)(x \bullet(y, z, t) k)(x, y z, t) k((x, y, z) k \bullet t) .
$$

In the second way also using Lemma 2.14(iii) to the term $((x, y) p \bullet z) \bullet t$, we get

$$
L=(x y z, t) p((x y, z) p \bullet t)(x y \bullet(z, t) p)^{-1}((x, y) p \bullet z t)(x y \bullet(z, t) p) .
$$

Using (3.27) to the first two terms and since $(x y, z, t) k \in \overline{\mathbf{Z}(\mathbf{A})}_{x y z t},(x, y, z t) k \in \overline{\mathbf{Z}(\mathbf{A})}_{x y z t}$, we finally get

$$
L=(x, y z t) p(x \bullet(y, z t) p)(x y \bullet(z, t) p)(x, y, z t) k(x y, z, t) k .
$$

Comparison gives

$$
(x y, z, t) k(x, y, z t) k=((x, y, z) k \bullet t)(x, y z, t) k(x \bullet(y, z, t) k) .
$$

Hence, by (3.14) $k$ is a 3 -cocycle.

Definition 3.4. The cocycle $k$ satisfying (3.27) is called an obstruction of the abstract kernel $\Psi: S \rightarrow \operatorname{Reg}_{E}(\mathbf{A}) / \operatorname{Inn}_{E}(\mathbf{A})$. The following lemma shows that the cohomology class defined by $k$ is independent of chosen $\sigma$ and $p$.

Lemma 3.5. (i) For a given $\sigma$, a change in the choice of $p$ in (3.17) replaces $k$ by a cohomologous cocycle. By suitably changing the choice of $p, k$ may be replaced by any cohomologous cocycle.

(ii) A change in the choice of $\sigma$ may be followed by a suitable new selection of $p$ so as to leave the obstruction cocycle $k$ unchanged.

Proof. (i) Suppose $p^{\prime}$ is another choice of $p$ and let $k^{\prime}$ be the corresponding 3-cocycle so that

$$
(x y, z) p^{\prime}\left((x, y) p^{\prime} \bullet z\right)=(x, y z) p^{\prime}\left(x \bullet(y, z) p^{\prime}\right)(x, y, z) k^{\prime}
$$

for all $x, y, z \in S$. We will show that $k, k^{\prime}$ are cohomologous. Since $p$ and $p^{\prime}$ satisfy (3.17), by Lemma 1.6, $((x, y) p) \eta=\left((x, y) p^{\prime}\right) \eta$. So the exactness of the sequence in Proposition 2.5 gives rise to a 2 -cochain $\tau: S \times S \rightarrow \overline{\mathbf{Z}(\mathbf{A})}$ such that

$$
(x, y) p^{\prime}=(x, y) p(x, y) \tau .
$$

Substituting (3.34) in (3.33) and using (3.17), we get

$$
(x, y, z) k k^{\prime-1}=(x, y, z) k(x, y, z) k^{\prime-1}=(x \bullet(y, z) \tau)(x y, z) \tau^{-1}(x, y z) \tau((x, y) \tau \bullet z)^{-1}
$$

for all $x, y, z \in S$. Thus by (3.15) $k$ and $k^{\prime}$ are cohomologous. To prove the second statement, take any 3 -cocycle $k^{\prime}$ that is cohomologous to $k$. Then there is a 2-cochain $\tau$ : $S \times S \rightarrow \overline{\mathbf{Z}(\mathbf{A})}$ such that (3.35) holds. If we put $(x, y) p^{\prime}=(x, y) p(x, y) \tau, x, y \in S$, then $p^{\prime}$ satisfies (3.17) and (3.33), and so $k^{\prime}$ is the 3-cocycle defined by $p^{\prime}$. 
(ii) Let $\sigma^{\prime}: S \rightarrow \operatorname{Reg}_{E}(\mathbf{A})$ be another map such that $(x) \sigma^{\prime} \in(x) \Psi$ for all $x \in S$, and let $\circ$ denote the biaction of $S$ on $A$ induced by $\sigma^{\prime}$. Then by Lemma 2.11(ii) there exists a map $\beta: S \rightarrow \mathbf{A},(x) \beta \in A_{x}$, such that $(x) \sigma^{\prime}=(x) \sigma((x) \beta) \eta$ for all $x \in S$. This implies by Lemma 3.2

$$
\left(x \sigma^{\prime}\right)\left(y \sigma^{\prime}\right)=(x y) \sigma^{\prime}\left((x y) \beta^{-1}(x, y) p((x) \beta \bullet y)(x \bullet(y) \beta)\right) \eta .
$$

Put $(x, y) p^{\prime}=(x y) \beta^{-1}(x, y) p((x) \beta \bullet y)(x \bullet(y) \beta) \eta$. Then, by Lemma 2.11(i) and (ii),

$$
(x, y) p^{\prime}=(x y) \beta^{-1}(x, y) p(x \circ(y) \beta)((x) \beta \circ y) .
$$

By Lemma 2.14, (3.37), and by the relation $x \circ(y \circ(z) \beta=x y \circ(z) \beta)$, we have

$$
(x y z) \beta(x y, z) p^{\prime}\left((x, y) p^{\prime} \circ z\right)=(x y z) \beta(x, y z) p^{\prime}\left(x \circ(y, z) p^{\prime}\right)(x, y, z) k .
$$

Hence,

$$
(x y, z) p^{\prime}\left((x, y) p^{\prime} \circ z\right)=(x, y z) p^{\prime}\left(x \circ(y, z) p^{\prime}\right)(x, y, z) k .
$$

Thus the obstruction cocycle determined by $p^{\prime}$ coincides with $k$.

From Lemmas 3.3 and 3.5, we obtain the first part of the following.

Theorem 3.6. Let $\Psi: S \rightarrow \operatorname{Reg}_{E}(\mathbf{A}) / \operatorname{Inn}_{E}(\mathbf{A})$ be an abstract kernel. Then $\Psi$ defines a welldefined element $[k]$ of $\mathbf{H}^{3}\left(S^{I}, \breve{Z}(\mathbf{A})^{0} \mathbf{F}\right)$. Further, $\Psi$ has an extension of $S$ by $\mathbf{A}$ if and only if $[k]=0$.

Proof. If $\Psi$ has an extension, then by Theorem 2.18 there is an extension of the form $(S, \sigma, p, \mathbf{A})$ with abstract kernel $\Psi$ and crossed pair $(\sigma, p)$. Then, since $(\sigma, p)$ satisfies $(2.48)$, it is clear from (3.27) that $[k]=0$. Conversely, suppose $[k]=0$. In view of Lemma 3.5(i), we can assume without loss of generality that $k=0$, the zero 3 -cocycle. Then $(\sigma, p)$ is a crossed pair by (3.17) and (3.27), and the crossed extension $(S, \sigma, p, \mathbf{A})$ is an extension of $S$ by $\mathrm{A}$ with abstract kernel $\Psi$ by Theorems 2.15 and 2.16 .

Theorem 3.7. Let $(S, \sigma, p, \mathbf{A})$ and $(S, \sigma, q, \mathbf{A})$ be two crossed extensions of $S$ by $\mathbf{A}$ with $a b$ stract kernel $\Psi$. Then $(S, \sigma, p, \mathbf{A})$ is equivalent to $(S, \sigma, q, \mathbf{A})$ if and only if there exists an 1 - cochain $\beta: S \rightarrow \overline{\mathbf{Z}(\mathbf{A})}$ such that

$$
(x, y) p(x, y) q^{-1}=((x) \beta \bullet y)(x \bullet(y) \beta)(x y) \beta^{-1}
$$

for all $x, y \in S$.

Proof. Suppose $(S, \sigma, p, \mathbf{A})$ and $(S, \sigma, q, \mathbf{A})$ are equivalent extensions and let $\theta: T_{p} \rightarrow T_{q}$ be an isomorphism such that $\theta \pi_{q}=\pi_{p}$ and $(a) U_{p} \theta=(a) U_{q}, a \in \mathbf{A}_{e}, e \in E$. Define maps $j_{1}: S \rightarrow T_{p}$ and $j_{2}: S \rightarrow T_{q}$ by $(x) j_{1}=\left(x, 1_{x}\right) ;(x) j_{2}=\left(x, 1_{x}\right)$, where $1_{x}$ denotes the identity element of $\mathbf{A}_{x}$ for all $x \in S$. Then $j_{1} \pi_{p}=1_{S}=j_{2} \pi_{q}$. For $x, y \in S$ and by Lemma 2.13(i) and (ii), we can easily show that $(x y) j_{1}((x, y) p) U_{p}=\left(x j_{1}\right)\left(y j_{1}\right)$. That is, $\left(x j_{1}\right)\left(y j_{1}\right)=$ $(x y) j_{1}((x, y) p) U_{p}$. Similarly $\left(x j_{2}\right)\left(y j_{2}\right)=(x y) j_{2}((x, y) q) U_{q}$. The proof of Theorem 2.16 gives $(x) j_{1} \bar{\mu}_{1}=(x) \sigma(x) j_{2} \bar{\mu}_{2}$ for all $x \in S$, where $\bar{\mu}_{1}: T_{p} \rightarrow \operatorname{Reg}_{E}(\mathbf{A})$ and $\bar{\mu}_{2}: T_{q} \rightarrow \operatorname{Reg}_{E}(\mathbf{A})$ 
are defined by (2.16). If we denote the composite $j_{1} \theta: S \rightarrow T_{q}$ by $j$, then $j \pi_{q}=1_{S},(x j)(y j)$ $=(x y) j((x, y) p) U_{q},(x) \sigma=(x) j \bar{\mu}_{2}$ for all $x, y \in S$. Since $j \pi_{q}=1_{S}=j_{2} \pi_{q}$, by Lemma 1.6 there exists a map $\beta: S \rightarrow \mathbf{A},(x) \beta \in \mathbf{A}_{x}$, such that

$$
(x) j=(x) j_{2}((x) \beta) U_{q}
$$

and so $(x) \sigma=(x) \overline{j \mu}_{2}=(x) j_{2} \bar{\mu}_{2}((x) \beta) U_{q} \bar{\mu}_{2}=(x) \sigma((x) \beta) \eta$ for all $x \in S$. Then, by Lemma 1.6, $(x) \beta \in \operatorname{Ker} \eta$ or $((x) \beta) \eta=\left(1_{x}\right) \eta$ (where $1_{x}$ is the identity element of $\mathbf{A}_{x}$ ) and therefore by Proposition $2.5,(x) \beta \in \overline{\mathbf{Z}(\mathbf{A})_{x}}$ for all $x \in S$. Thus $\beta$ is a 1-cochain. By using Lemma 2.17 and $((x) \beta \bullet y)(x \bullet(y) \beta) \in \overline{\mathbf{Z}(\mathbf{A})}_{x y}$, we easily derive

$$
(x y) j=(x y) j\left[(x y) \beta^{-1}(x, y) q(x, y) p^{-1}((x) \beta \bullet y)(x \bullet(y) \beta)\right] U_{q} .
$$

Then, by Lemma 1.6, $(x y) \beta^{-1}(x, y) q(x, y) p^{-1}((x) \beta \bullet y)(x \bullet(y) \beta)=1_{x y}$ or, since $\beta$ takes values in $\overline{\mathbf{Z}(\mathbf{A})}$,

$$
(x, y) p(x, y) q^{-1}=((x) \beta \bullet y)(x \bullet(y) \beta)(x y) \beta^{-1} .
$$

Conversely, let $\beta: S \rightarrow \overline{\mathbf{Z}(\mathbf{A})}$ be a 1-cochain such that (3.40) holds. This implies, in particular, $(x) \beta$ commutes with every element of $\mathbf{A}_{x}, x \in S$. Define a map $\theta: T_{p} \rightarrow T_{q}$ by $(x, a) \theta=(x, a(x) \beta)$ for all $(x, a) \in T_{p}$. Then clearly $\theta \pi_{q}=\pi_{p}$. Moreover, for $e \in E(S)$ and $a \in \mathbf{A}_{e}$, by (2.65) we get $(a) U_{p} \theta=\left(e,(e, e) p^{-1} a\right) \theta=\left(e,(e, e) p^{-1}(e) \beta a\right)=\left(e,(e, e) q^{-1} a\right)=$ (a) $U_{q}$ since (3.40) implies $(e, e) q^{-1}=(e, e) p^{-1}(e) \beta \bullet e$, and by Lemma 2.13(i), $(e) \beta \bullet e=$ (e) $\beta$. Using (3.40) we can easily verify $\theta$ is an isomorphism. Hence, $(S, \sigma, p, \mathbf{A})$ and $(S, \sigma$, $q, \mathbf{A})$ are equivalent.

TheOREM 3.8. If the abstract kernel $\Psi: S \rightarrow \operatorname{Reg}_{E}(\mathbf{A}) / \operatorname{Inn}_{E}(\mathbf{A})$ has an extension, then the set $\varepsilon(S, \mathbf{A})$ of equivalence classes of extensions of $S$ by $\mathbf{A}$ with abstract kernel $\Psi$ is in one-to-one correspondence with the set $\mathbf{H}^{2}\left(S^{I}, \check{\mathbf{Z}}(\mathbf{A})^{0} \mathbf{F}\right)$.

Proof. Since $\Psi$ admits an extension of $S$ by A, by Theorem 2.18, there is an extension of the form $(S, \sigma, p, \mathbf{A})$ with abstract kernel $\Psi$. Keep $\sigma$ fixed. Let $\alpha: S \times S \rightarrow \overline{\mathbf{Z}(\mathbf{A})}$ be a 2 -cocycle so that $(x y, z) \alpha((x, y) \alpha \bullet z)=(x, y z) \alpha(x \bullet(y, z) \alpha)$ for all $x, y, z \in S$. Define $p \alpha$ : $S \times S \rightarrow A$ by $(x, y) p \alpha=(x, y) p(x, y) \alpha$. Then $(\sigma, p \alpha)$ is a crossed pair and hence defines a crossed extension $(S, \sigma, p \alpha, \mathbf{A})$ with abstract kernel $\Psi$. If $\alpha^{\prime}$ is another 2-cocycle, then

$$
\begin{aligned}
(x, y) \alpha^{-1}(x, y) \alpha^{\prime} & =(x, y) \alpha^{-1}(x, y) p^{-1}(x, y) p(x, y) \alpha^{\prime} \\
& =\left((x, y) p(x, y) \alpha^{-1}\right)(x, y) p(x, y) \alpha^{\prime} \\
& =((x, y) p \alpha)^{-1}(x, y) p \alpha^{\prime} .
\end{aligned}
$$

Therefore, using Theorem 3.7, it is easy to see that $\alpha, \alpha^{\prime}$ are cohomologous if and only if $(S, \sigma, p \alpha, \mathbf{A})$ and $\left(S, \sigma, p \alpha^{\prime}, \mathbf{A}\right)$ are equivalent. Hence, we have a well-defined injective map

$$
\xi:[\alpha] \longrightarrow[S, \sigma, p \alpha, \mathbf{A}]: \mathbf{H}^{2}\left(S^{I}, \check{\mathbf{Z}}(\mathbf{A})^{0} \mathbf{F}\right) \longrightarrow \varepsilon(S, \mathbf{A})
$$


where $[S, \sigma, p \alpha, \mathbf{A}]$ denotes the equivalence class of $(S, \sigma, p \alpha, \mathbf{A})$. Let $(S, \sigma, q, \mathbf{A})$ be an extension of $S$ by A with abstract kernel $\Psi$. Then by (2.23), Lemma 1.6, and Proposition 2.5, we prove $(x, y) q(x, y) p^{-1} \in \mathbf{Z}(\mathbf{A})_{(x y) * x y}=\overline{\mathbf{Z}(\mathbf{A})}_{x y}$. Put $(x, y) \alpha=(x, y) q(x, y) p^{-1}$. Then $\alpha: S \times S \rightarrow \overline{\mathbf{Z}(\mathbf{A})},(x, y) \alpha \in \overline{\mathbf{Z}(\mathbf{A})_{x y}}$ is a 2-cochain. $\alpha$ is indeed a 2-cocycle. So $[\alpha] \in \mathbf{H}^{2}\left(S^{I}\right.$, $\left.\check{\mathbf{Z}}(\mathbf{A})^{0} \mathbf{F}\right)$ and $[\alpha] \xi=[S, \sigma, p \alpha, \mathbf{A}]=[S, \sigma, q, \mathbf{A}]$. Since every extension of $S$ by $\mathbf{A}$ with abstract kernel $\Psi$ is equivalent to an extension of the form $(S, \sigma, q, \mathbf{A})$ by Theorem 2.18 , it follows that $\xi$ is surjective. The proof of the theorem is complete.

Theorems 3.6 and 3.8 generalize the corresponding results for inverse semigroups due to Lausch [4].

\section{References}

[1] A. H. Clifford and G. B. Preston, The Algebraic Theory of Semigroups. Vol. I, Mathematical Surveys, no. 7, American Mathematical Society, Rhode Island, 1961.

[2] S. Eilenberg and S. Mac Lane, Cohomology theory in abstract groups. II. Group extensions with a non-Abelian kernel, Ann. of Math. (2) 48 (1947), 326-341.

[3] G. Lallement, Demi-groupes réguliers, Ann. Mat. Pura Appl. (4) 77 (1967), 47-129 (French).

[4] H. Lausch, Cohomology of inverse semigroups, J. Algebra 35 (1975), no. 1-3, 273-303.

[5] J. Leech, The D-category of a semigroup, Semigroup Forum 11 (1975/76), no. 4, 283-296.

[6] - The cohomology of monoids, preprint, 1987.

[7] M. Loganathan, Complementation and inner automorphism for regular semigroups, Semigroup Forum 21 (1980), no. 2-3, 195-204.

[8] _ Cohomology of inverse semigroups, J. Algebra 70 (1981), no. 2, 375-393.

[9] Cohomology and extensions of regular semigroups, J. Austral. Math. Soc. Ser. A 35 (1983), no. 2, 178-193.

[10] M. Loganathan and A. Tamilarasi, Construction and representation of regular semigroups, Semigroup Forum 51 (1995), no. 2, 191-216.

[11] S. Mac Lane, Homology, Die Grundlehren der mathematischen Wissenschaften, vol. 114, Academic Press, New York; Springer, Berlin, 1963.

[12] - Categories for the Working Mathematician, Graduate Texts in Mathematics, vol. 5, Springer, New York, 1971.

[13] K. S. S. Nambooripad, Structure of regular semigroups. I, Mem. Amer. Math. Soc. 22 (1979), no. 224 , vii+119.

[14] F. J. Pastijn and M. Petrich, Regular Semigroups as Extensions, Research Notes in Mathematics, vol. 136, Pitman, Massachusetts, 1985.

[15] M. Petrich, Extensions normales de demi-groupes inverses, Fund. Math. 112 (1981), no. 3, 187203 (French).

A. Tamilarasi: Department of Mathematics, Kongu Engineering College, Perundurai 638052, Tamil Nadu, India

E-mail address: a_tamilarasi@yahoo.com 


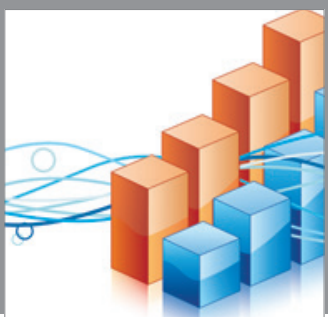

Advances in

Operations Research

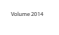

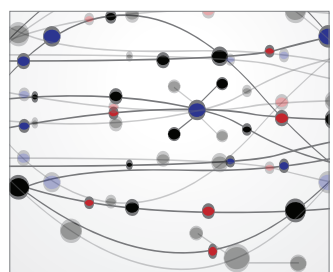

\section{The Scientific} World Journal
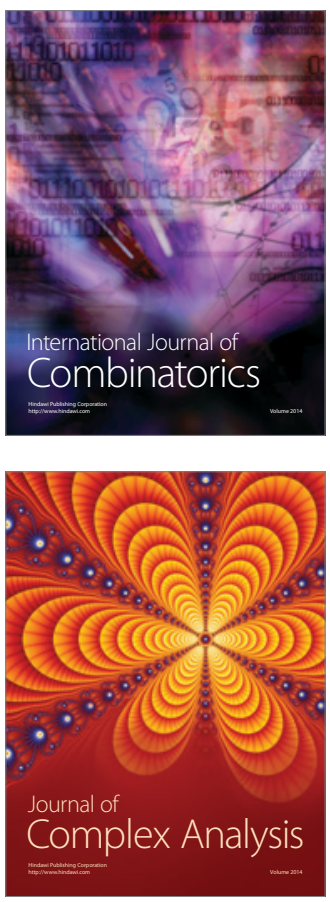

International Journal of

Mathematics and

Mathematical

Sciences
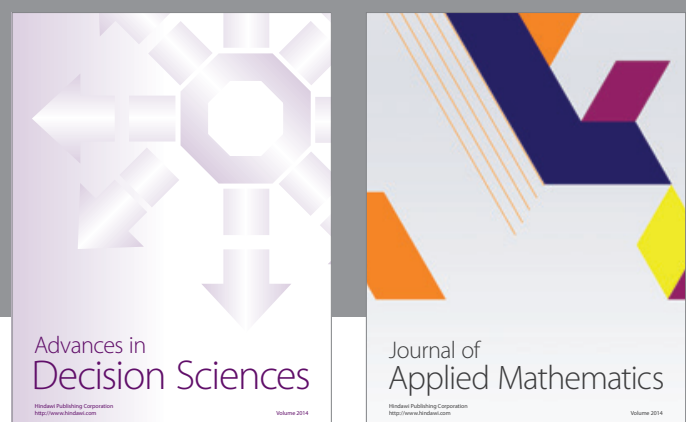

Journal of

Applied Mathematics
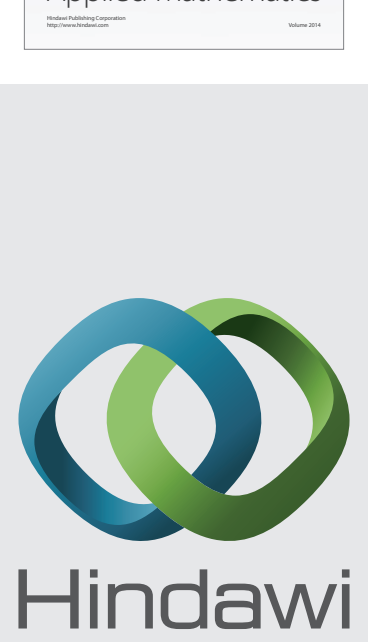

Submit your manuscripts at http://www.hindawi.com
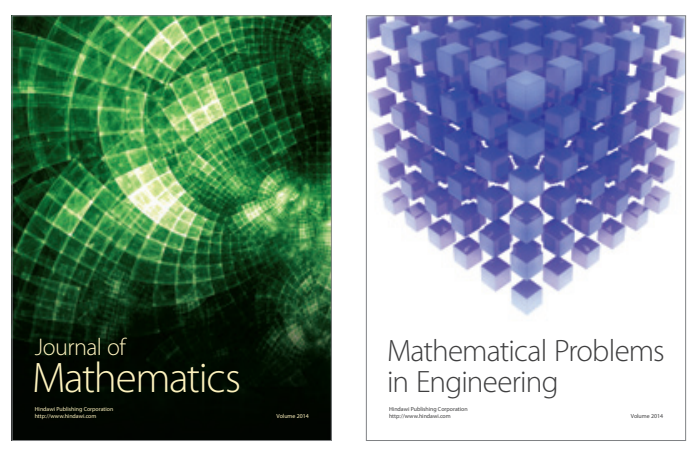

Mathematical Problems in Engineering
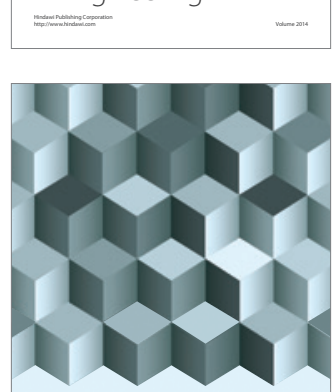

Journal of

Function Spaces
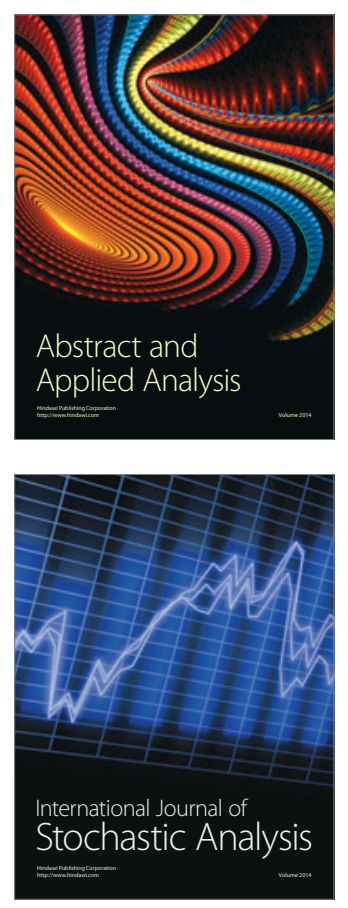

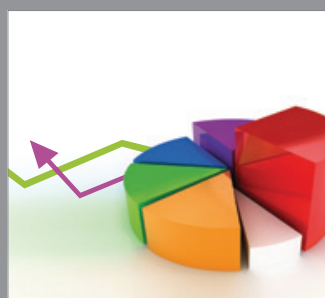

ournal of

Probability and Statistics

Promensencen
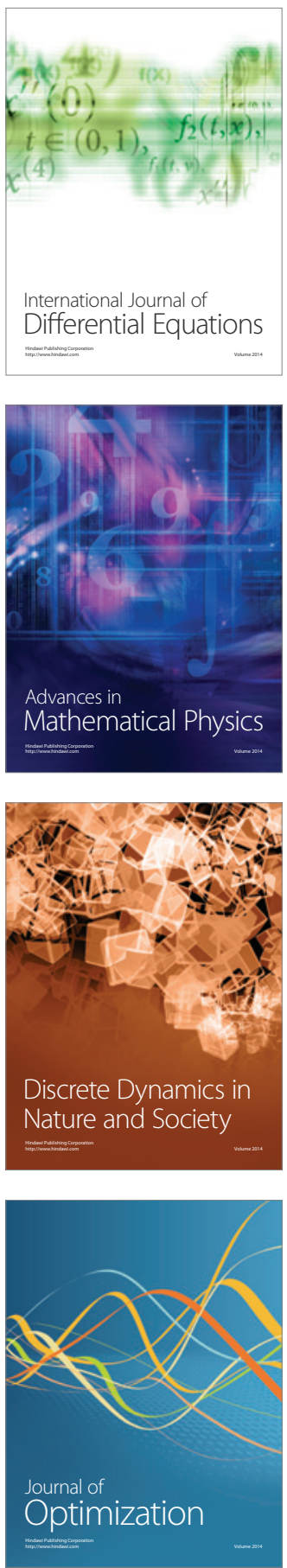